\title{
ArcheoSciences
}

Revue d'archéométrie

\section{Une base pour l'élaboration de modèles de peuplement de l'Âge du Fer en France}

A Basis for the development of settlement pattern of the Iron Age in France

Olivier Buchsenschutz, Christophe Batardy, Michel Cartereau, Katherine Gruel et Marc Levéry

\section{(2) OpenEdition}

12 Journals

Édition électronique

URL : https://journals.openedition.org/archeosciences/4457

DOI : $10.4000 /$ archeosciences. 4457

ISBN : 978-2-7535-4778-0

ISSN : $2104-3728$

Éditeur

Presses universitaires de Rennes

Édition imprimée

Date de publication : 31 décembre 2015

Pagination : 157-175

ISBN : 978-2-7535-4776-6

ISSN : $1960-1360$

\section{Référence électronique}

Olivier Buchsenschutz, Christophe Batardy, Michel Cartereau, Katherine Gruel et Marc Levéry, « Une

base pour l'élaboration de modèles de peuplement de l'Âge du Fer en France », ArcheoSciences [En

ligne], 39 | 2015, mis en ligne le 31 décembre 2017, consulté le 27 janvier 2022. URL : http://

journals.openedition.org/archeosciences/4457 ; DOI : https://doi.org/10.4000/archeosciences. 4457 


\title{
Une base pour l'élaboration de modèles de peuplement de l'Âge du Fer en France
}

\author{
A Basis for the Development of Settlement Pattern of the Iron Age in France
}

\author{
Olivier Buchsenschutz ${ }^{a}$, Christophe Batardy ${ }^{\mathrm{b}}$, Michel Cartereau ${ }^{c}$, \\ Katherine Gruel ${ }^{\mathrm{d}}$ et Marc LeVÉRY ${ }^{\mathrm{d}}$
}

\begin{abstract}
Résumé : Nous avons développé une base de données géoréférencées pour faire des analyses statistiques et spatiales. 15000 gisements l'Âge du Fer ont été répertoriés sur le territoire national à partir de thèses, d'articles thématiques et de la collection "Carte archéologique de la Gaule ". Les premières statistiques offrent un point de vue inédit sur cent cinquante ans de recherche. La répartition des gisements documentés par leur fonction et leur mobilier permet de proposer sinon des modèles de peuplement, du moins des variations chronologiques et régionales à valeur historique, libérées à cette échelle des biais de la prospection archéologique.
\end{abstract}

\begin{abstract}
We have developed a geo-referenced database for statistical and spatial analysis. 15.000 deposits from the Iron Age have been listed on the national territory from theses, thematic articles and the collection "Carte archéologique de la Gaule". The first statistics offer an unique perspective a hundred fifty years of research. The distribution of it not deposits documented by their functions and assemblage initial is used to give models of settlement patterns, at least of chronological and regional variations of historical value, released on this scale from the bias of the archaeological survey.
\end{abstract}

Mots clés : Âge du Fer, France, base de données, statistique, analyse spatiale, chronologie, modèles de peuplement.

Keywords: Iron Age, France, database, statistics, spatial analysis, chronology, settlement pattern.

\section{INTRODUCTION}

Les tentatives de cartographie systématique du territoire français à l'Âge du Fer sont restées rares ou à l'état de manuscrit. La collection de la "Carte archéologique de la Gaule", qui couvre aujourd'hui l'ensemble du territoire, réunit la bibliographie et des notices sur les gisements antiques, y compris ceux de l'Âge du Fer dans les volumes récents (Provost et al., 1988-2013). C'est sur cette source et un cer- tain nombre de thèses ou d'articles thématiques ${ }^{1}$ que nous nous sommes appuyés pour constituer une base de données géo-référencées et dresser des cartes et des statistiques dont nous présentons ici quelques exemples.

L'objectif de cette enquête est de dresser un état des lieux et de développer un outil de travail, à un moment où le nombre de données récoltées sur l'Âge du Fer en France augmente dans des proportions analogues à celles des publications de la fin du XIX ${ }^{e}$ s., qui servaient encore de base de

a Olivier BUCHSENSCHUTZ (CNRS) UMR 8546 Archéologie et philologie d'Orient et d'Occident, ENS 45, rue d'Ulm F 75230 Paris Cedex 05. (buchs@ ens.fr)

${ }^{b}$ Éducation Nationale, CNRS UMR 8546.

'AgroParisTech - 16 rue Claude Bernard F-75231 Paris Cedex 05 Tel : 33 (0) 1440818 43. (michel.cartereau@agroparistech.fr)

${ }^{d}$ CNRS UMR 8546 (katherine.gruel@ens.fr)

1. Nous ne pouvons pas citer ici toutes ces références (Nash, Freidin, Fichtl, Bocquet, Mohen, Roulière etc.) qui comprennent des synthèses publiées essentiellement de 1980 à aujourd'hui, des maîtrises et des thèses. Le dépouillement des Cartes Archéologiques de la Gaule n'est bien sûr qu'une base de départ. 
réflexion dans les années 1950. Les critères descriptifs retenus dans les fiches permettent déjà de dresser des statistiques par thème, par région, par période. Nous avons délibérément orienté l'analyse en vue des analyses spatiales et des cartographies thématiques. Il ne s'agit pas en effet ici de répondre à la question : "qu'y a-t-il à tel endroit? ", mais plutôt " où sont toutes les [fortifications, incinérations, fibules...] de telle période? " Les méthodes de construction de ces cartes ont été définies depuis longtemps (Eggers, 1951), mais les protohistoriens qui ont travaillé sur l'espace ont privilégié des échelles plus grandes et l'analyse des relations hommemilieu, plutôt que des répartitions complexes d'artefacts à petite échelle, avant ces dernières décennies (Buchsenschutz et al., 2004). Il nous a fallu dix ans pour réunir un premier lot de données cohérent et pour trouver un équilibre entre une impossible base universelle et un annuaire sec et stérile. À cette échelle de travail (la base est pertinente de l'échelle régionale à l'échelle nationale ou européenne), les biais de la prospection ou de la densité des fouilles disparaissent, les répartitions obtenues prennent une valeur " historique " et peuvent être modélisées. Nous rappellerons brièvement les principales caractéristiques de la Basefer, avant d'analyser par différentes méthodes sa pertinence pour montrer l'évolution chronologique et spatiale du peuplement de l'hexagone à l'Âge du Fer. Ensuite l'exemple des monnaies montrera ce qu'on peut tirer d'une analyse thématique.

\section{ObJeCtifs ET ORGANISATION DE LA BASEFER}

La «Basefer » a déjà été présentée en 2008 (Batardy et al.), alors que tous les départements n'étaient pas encore couverts. Pour les détails sur sa structure et son but, nous prions le lecteur de bien vouloir se référer à cet article.

\section{Objectifs}

La BaseFer a pour principal objectif de visualiser rapidement l'état des connaissances pour l'Âge du Fer sur le territoire métropolitain. Elle est destinée à établir des cartes de répartition après un traitement statistique des données. Elle se limite actuellement à des gisements publiés et interprétés².

2. Nous envisageons l'introduction de données issues de rapports de fouille ou de prospection, mais dans un second temps, et lorsqu'ils seront facilement accessibles, comme c'est déjà le cas pour la Bretagne. Nous développons actuellement une méthode de codification automatique. Nous ne souhaitons pas toutefois intégrer.les données de rapports qui n'ont pas été clairement analysées et interprétées par l'auteur des travaux de terrain.
Une fiche correspond à un seul site ayant la même fonction dans une occupation continue. Les données restent essentiellement qualitatives : la surface des habitats ou le nombre d'objets peut être signalé en commentaire, mais il ne fera pas l'objet de calculs systématiques. De même la stratigraphie fine, l'organisation des sépultures individuelles ou les structures d'habitat ne sont pas prises en compte.

Les critères descriptifs ont été retenus lorsqu'on estimait qu'ils seraient représentés dans la base par 50 à 500 individus pour que leur répartition soit significative (ex. "fibule ", " fusaïole », « ossement animal») (fig. 1a). Des données plus fines, ou qui demandent l'intervention d'un spécialiste (type de fibule, identification d'ossement), ne sont pas prises en compte.

La base doit servir à documenter le contexte général de l'Âge du Fer dans lequel s'inscrivent des bases plus spécialisées, qui la complètent, comme les bases sur les ossements animaux, les meules, les plantes, ou par exemple les bases élaborées par K. Gruel sur les monnaies et les faciès monétaires. Elle ne prétend pas se substituer à elles. Elle vise à prendre en charge les contextes (administratif, topographique, archéologique, chronologique) et à faciliter les comparaisons entre elles.

\section{L'organisation de la BaseFer}

\section{Organisation informatique}

La géolocalisation d'un site s'appuie sur les coordonnées de sa commune, et si besoin, la base permet d'enregistrer des coordonnées plus précises en spécifiant un référentiel géographique. La base complémentaire Archeolocalis assure la description des communes avec l'indication de ses centroïdes dans le référentiel mondial WGS84 (world geodesic system 1984) et dans un référentiel national, et avec la prise en compte des niveaux administratifs, dans une dimension internationale (fig. 1b).

Le modèle de données de la base Basefer (fig. 1b) s'appuie donc sur celui d'Archeolocalis au niveau de la localisation du site. Sa complexité principale réside dans la description des différentes structures, plus précisément le rattachement entre les trois niveaux de structures détaillées et les structures principales.

L'architecture logicielle déployée repose principalement sur un stockage des données centralisé en SQL (système MySQL actuellement), l'utilisation de Filemaker pour la saisie des données, et une application spécifique en ligne (réalisée en PHP et javascript) pour l'interrogation de la base sur un modèle où/quand/quoi, l'exportation de données et la réalisation de comptages. Par ailleurs, l'identifiant de gise- 
STRUCTURE STRUCTURE PRINCIPALE

Struct. 1

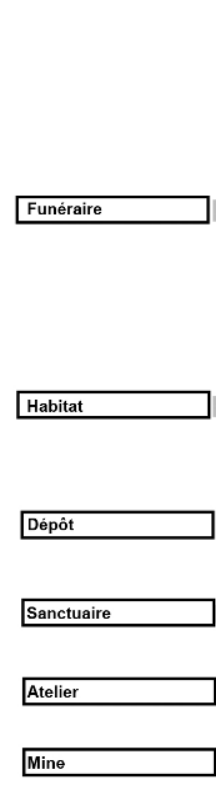

MOBILIER
Struct. 1

Cimetière tombes plates

Tombe isolée

\begin{tabular}{|l|}
\hline Struct. 1 \\
Habitat isolé \\
Habitat fortifié \\
Habitat groupé \\
Grotte \\
\hline
\end{tabular}

Grotte

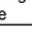

Struct. 2

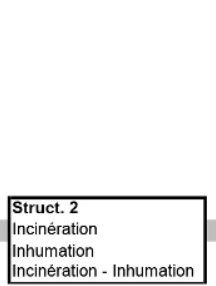

Struct.2

Artisanat

Enclos

Fortification
Structure constru

Structure construite
Structure en creux

Voirie

Struct. 3

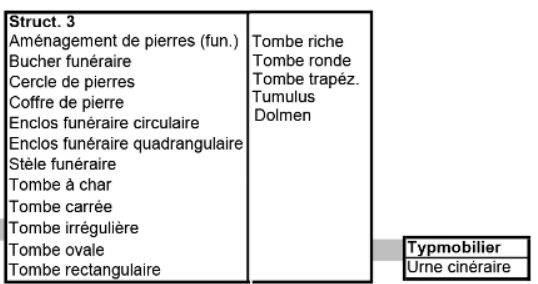

Tombe rectangulaire

\begin{tabular}{|c|c|c|}
\hline \multirow[b]{2}{*}{\begin{tabular}{|l|} 
Struct. 3 \\
Aménagement de pierres (hab.) \\
Bâtiment en pierre \\
Bâtiment en terre \\
Bâtiment mixte \\
Bâtiment sur poteaux porteurs \\
Bâtiment sur solin-sablière \\
Cave \\
Construction circulaire \\
Construction rectangulaire \\
Enclos circulaire \\
Enclos quadrangulaire
\end{tabular}} & & \\
\hline & $\begin{array}{l}\text { Fosse (la) } \\
\text { Fossé(le) } \\
\text { Fosse circulaire } \\
\text { Four } \\
\text { Foyer } \\
\text { Grenier } \\
\text { Mare } \\
\text { Murus gallicus } \\
\text { Mur pierre } \\
\text { Mur terre } \\
\text { Palissade } \\
\text { Pilotis }\end{array}$ & $\begin{array}{l}\text { Puits } \\
\text { Rempart } \\
\text { Rempart à poutrage } \\
\text { Rempart calciné } \\
\text { Rempart en pierres sèches } \\
\text { Rempart massif } \\
\text { Rempart vitrifié } \\
\text { Silo } \\
\text { Souterrain } \\
\text { Stele en réemploi } \\
\text { Talus en } \\
\text { Torchis } \\
\text { Tour } \\
\text { Trou de poteau }\end{array}$ \\
\hline
\end{tabular}

Enclos quadrangulaire Pilotis

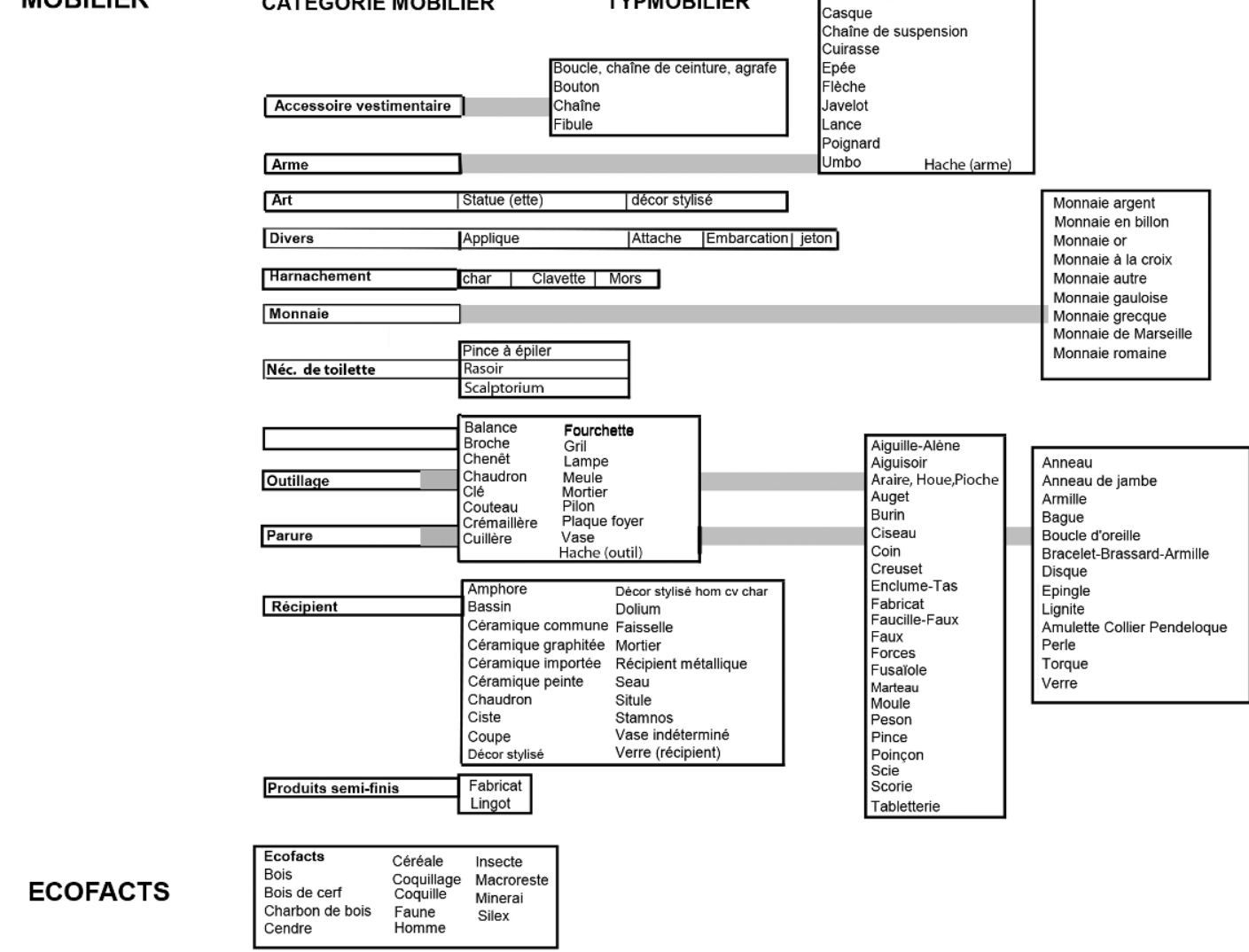

Figure 1 : a) Structuration de la description des gisements dans la Basefer. b) modèle de données de la base Archeolocalis [modele-archeolocalis-GMPCA.png] Data structuration of the Database Archeolocalis. (La notation des modèles de base présentés ici correspond au modèle entité-association.) c) modèle de données de la base Basefer Data structuration of the Database basefer.

Figure 1: Structuring of the deposit's description in the Basefer 
b

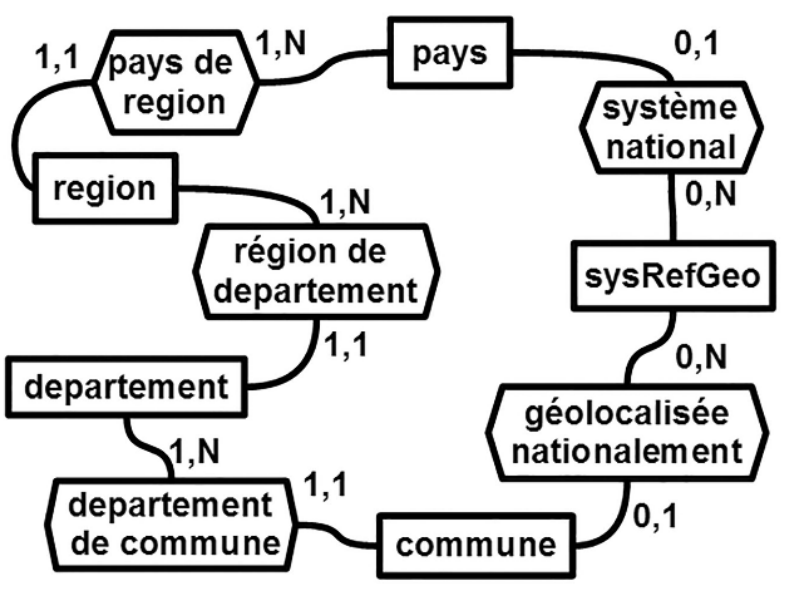

MC - 28 VII 2015

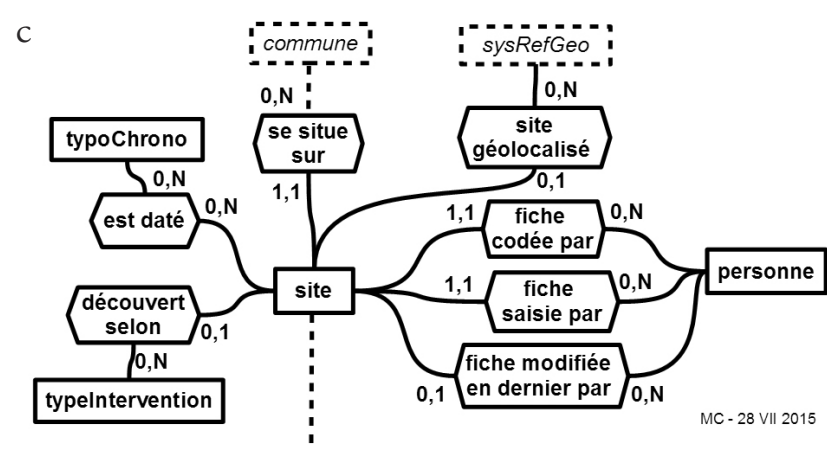

Figure 1 (suite)

Figure 1 (following) ment permet de relier la BaseFer à des bases plus spécialisées ou tout autre contenu plus approfondi. (figure 1c). Il existe une version en ligne de la BaseFer simplifiée et accessible au grand public ${ }^{3}$.

\section{Codage et interrogation}

Pour l'utilisateur la géolocalisation (Archeolocalis) est renseignée en même temps que la description des gisements (BaseFer) (figure 1). La datation est précisée par la chronotypologie et éventuellement par une datation absolue. Les structures (architectures, constructions diverses) sont définies selon trois niveaux, qui correspondent soit à une description hiérarchique, soit à différents aspects d'un domaine. Par exemple on trouvera pour les données funéraires :

Structure principale $=$ funéraire

str. 1 = tombe plate, tumulus, etc.

str. 2 = inhumation, incinération etc.

str. 3 = coffre de pierre, tombe à char etc.

Les "structures principales " sont volontairement très générales (habitat - 5733, funéraire - 4639, dépôt - 4686, objet isolé -621 , atelier -346 , sanctuaire -337 , parcellaire $\left.-35^{4}\right)$, nous verrons qu'elles sont très utiles pour caractériser périodes et régions.

3. Cet "Atlas de l'Âge du Fer" a pour objectif de permettre au public de se faire rapidement une idée sur l'état de la connaissance sur un pays, une région, une sous-région ou département $=$ Où?, à une période donnée $=$ Quand ?, à propos d'un thème et d'un sous-thème = Quoi ? Atlas de l'Âge du Fer, AOROC, [http://www.chronocarto.ens.fr/gcserverlatlas]. Le dépôt de ces cartes thématiques dans l'atlas se fait par l'intermédiaire de Chronocarto, le visualiseur en réseau de données géo-référencées d'AOROC, développé par la société Géocarta.

4. Les chiffres représentent les scores actuels; il est clair que " parcellaire » va être éliminé parce qu’il n’est pas représentatif.
Le mobilier récolté (Catégorie $=$ arme, parure, etc. et Type = fibule, bouterolle etc.) se limite aux grands marqueurs, sous forme de présence/absence. Cette subdivision simple permet de faire la carte des parures en général aussi bien que celle d'un type significatif.

Le logiciel interdit les synonymes et relie les niveaux de structure les uns aux autres. Il autorise plusieurs réponses par colonne, ce qui permet de cartographier aussi bien des sites très riches, comme une nécropole, que des objets isolés, et également de faire des calculs sur les associations d'objets. Les bases spécialisées sur les fibules, les meules, les armes etc., ne nous renseignent pas en général sur les associations d'objets, alors que la Basefer en revanche répond instantanément aux questions concernant les associations les plus variées, grâce à cette structuration. Il est alors possible de définir et de cartographier des caractéristiques culturelles générales (associations atelier-habitats, sanctuaire-dépôt monétaire, nécropoles-armes et parures etc.) par période et par région, reflétant un type de peuplement. L'étape suivante, la codification des types précis d'objets ou de structure (fibule de Nauheim, « murus gallicus»), qui permettra d'identifier plus précisément ces peuplements, reste embryonnaire tant que ces types ne sont pas entérinés par les spécialistes.

Des informations optionnelles donnent enfin quelques compléments d'information sur le contexte de découverte, la bibliographie, la création et l'évolution des fiches etc. 


\section{L'ÉTAT DES CONNAISSANCES SUR L'ÂGE du Fer en France en 2015 peUt-IL AUTORISER LA CONSTRUCTION DE MODÈLES DE PEUPLEMENT ?}

Les « protohistoriens » ont pris l'habitude, en l'absence de textes, de construire leurs hypothèses sur les peuplements à partir de la répartition d'un ou de plusieurs objets, puis de la développer en analysant les habitats, les rituels, l'organisation du territoire quand la masse de données le permettait (Buchsenschutz, 2006). La Basefer complète cette démarche et prétend la valider en replaçant ces signaux discrets d'une mode ou d'une pratique propre à une population particulière dans un contexte culturel général, complet : habitat, rituels, production, échanges. Voyons si les quelques 15000 gisements inventoriés permettent de faire cette validation, ou si les aléas de la conservation des sites et de l'inégalité de la recherche l'interdisent encore.

\section{La répartition générale des gisements}

Depuis la fin de l'année 2013, tous les départements ont été explorés, au moins à travers le volume que la Carte archéologique de la Gaule leur a consacré. La carte générale de l'ensemble des gisements ${ }^{5}$ est bien remplie. Par rapport aux cartes anciennes, les concentrations des ensembles funéraires du Nord-Est au Sud-Ouest subsistent, mais seulement pour certaines périodes. L'ensemble du territoire est occupé, sauf des régions dont le sol est peu favorable à une implantation humaine comme les zones marécageuses, ou les Alpes et les autres sommets montagneux (fig. 2).

Le fait que la carte soit globalement équilibrée, comme le montre la position du centre médian est en soi un résultat ${ }^{6}$, d'autant plus intéressant que les groupes culturels qui sont installés sur le fameux hexagone français sont, à l'Âge du Fer, étroitement apparentés. Il n'y a pas, comme par exemple dans les îles Britanniques ${ }^{7}$, des adaptations locales à un

5. Nous avons choisi le terme de " gisement " plutôt que celui de " site " pour désigner une découverte archéologique. Il permet de recouvrir aussi bien la fouille partielle ou totale d'une occupation humaine que la découverte d'un objet isolé mais retenu parce qu'il est exceptionnel. Le même flou existe en anglais et en allemand avec les termes de Settlement, Siedlung, Befunde...

6. Le centre médian : "Tandis que l'outil Centre moyen renvoie un point à la coordonnée $\mathrm{X}$ moyenne et à la coordonnée $\mathrm{Y}$ moyenne de tous les centroïdes des entités, le centre médian utilise un algorithme itératif pour identifier le point qui minimise la distance euclidienne vers toutes les entités du jeu de données " Arcmap.

7. La carte des fortifications met en évidence la relation entre leur nature et leur surface avec le contexte géographique (ouest, centre, et est de l'île) (Ordnance survey 1962).

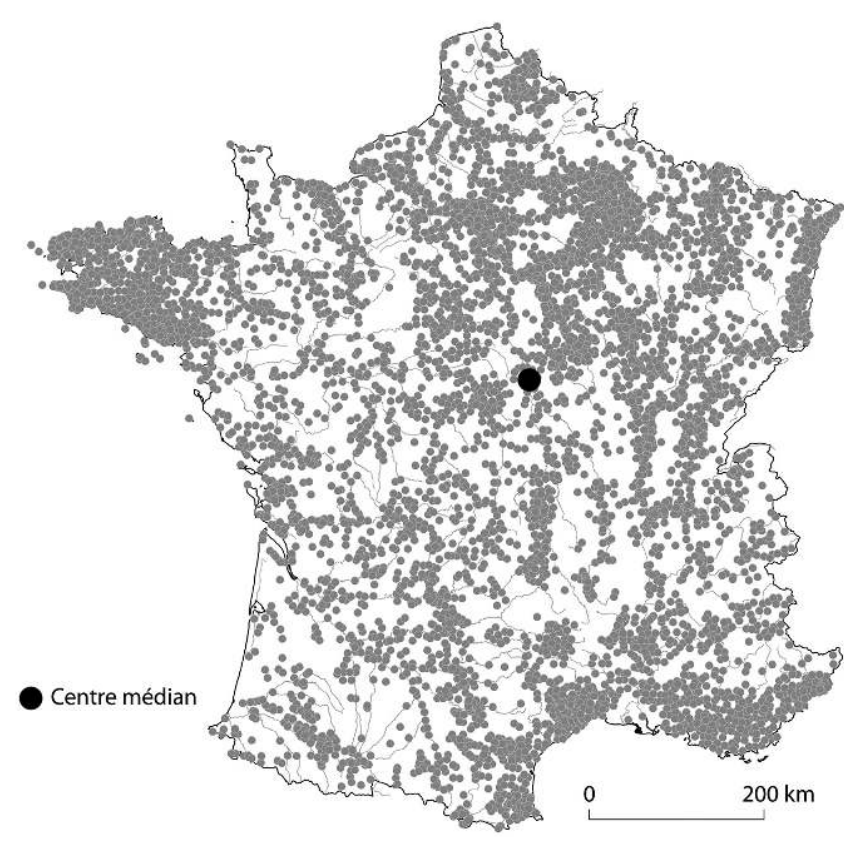

Figure 2 : Répartition et centre médian des gisements de la BaseFer en 2014, excepté les dépôts monétaires et les monnaies isolées. BaseFer mars 2014 (C).

Figure 2: Distribution and median Center of settlements of the BaseFer in 2014, except coins.

milieu naturel contraignant ou des différences culturelles fortes d'une région à l'autre.

L'information n'est pas, à cette petite échelle, fortement affectée par les conditions de conservation ou de fouille, comme les axes fluviaux, les zones cultivées favorables à la fois aux établissements humains et à la prospection archéologique aérienne ou de surface, les réseaux et les zones d'aménagement où l'archéologie de sauvetage a multiplié les occasions de découverte. L'outil qui résulte de cette enquête est pertinent surtout à petite échelle, il vise à mettre en évidence des tendances générales par grandes régions et par périodes. Mais il faut être plus prudent dès qu'on fait un "zoom " sur une région ou un département : il est alors nécessaire, avant d'interpréter la répartition des gisements, de faire une analyse critique des conditions de découverte pour corriger la répartition obtenue en fonction de ce masque déformant ${ }^{8}$. Même à petite échelle, la densité des gisements est beaucoup plus forte en Limagne que dans les

8. On trouvera un exemple de cette démarche dans Batardy et al., l'Atlas $d u$ Berry, 2000, p. 30. Il est possible de faire des cartes à valeur historique qui sont corrigées par la mise en évidence de ces masques, malgré l'idée encore répandue que la carte archéologique n'est que la carte des chercheurs. 
montagnes voisines, ou dans la plaine alsacienne que dans les Vosges, mais cela tient à la fois du nombre des opérations archéologiques de sauvetage, liées aux travaux actuels, et de la réalité de l'occupation humaine.

L'analyse spatiale permet, pour ceux qui sont plus sensibles à un calcul mathématique qu'à la perception de l'espace sur une carte, de vérifier notre impression visuelle. On constate que le centre médian est, dans l'état actuel du fichier, à quelques kilomètres du centre géographique de la France (fig. 2). Il est possible d'affiner ce résultat, soit en discutant sur le rôle de la côte méditerranéenne, de la Bretagne, de l'Alsace, des plaines du Bassin parisien, qui contribuent à cet équilibre pour des raisons différentes, soit en regardant comment ce centre médian se déplace suivant les thèmes ou les périodes. Quand on en reste aux grandes catégories (habitat, gisements funéraires, dépôts etc.), il varie d'environ $200 \mathrm{~km}$ au maximum. Pour des objets particuliers, comme les meules à céréales par exemple, il peut varier beaucoup plus selon les périodes en fonction des recherches, régionales et dispersées, sur cette catégorie d'objets (fig. 3).

La carte de répartition ponctuelle des gisements peut être utilement complétée par une carte de densité, qui rend mieux compte des fortes concentrations (fig. 4). Ici le territoire a été découpé en carrés de 50 x $50 \mathrm{~km}$, occupant une surface de $2500 \mathrm{~km}^{2}$. Aucune zone n'est totalement vide ${ }^{10}$, mais l'écart est grand entre celles qui comptent moins d'une vingtaine de gisements, et la Limagne ou certaines zones de l'est du Bassin parisien qui peuvent en réunir jusqu'à 250 , soit une moyenne de 1 gisement pour $10 \mathrm{~km}^{2}$. La Bretagne, l'Alsace, le centre du Bassin parisien, la Bourgogne, le Languedoc oriental et la Provence sont les zones les plus denses avec plus de 100 gisements pour $2500 \mathrm{~km}^{2}$. La carte globale présentée ci-dessous ne sert qu’à mettre en évidence la densité générale de l'occupation du territoire. Bien que les détails à grande échelle soient ici gommés, les conditions de la recherche influencent encore la répartition des sites. Il n'y a pas de raison " historique " pour que la densité des sites dans le quart nord-est de l'Île-de-France ou en Provence centrale soit aussi forte. Il faudra donc systématiquement comparer cette répartition globale avec la distribution d'un

9. Les résultats de l'enquête nationale en cours du "Groupe Meule ", qui elles-mêmes ne couvrent pas encore tout le territoire de façon systématique, n'ont pas été prises en compte dans la base. Les participants à ce groupe ont été très surpris de la carte obtenue ici, à partir essentiellement des citations trouvées dans la Carte archéologique de la France. L'examen des résultats obtenus sur les amphores a aussi fortement motivé F. Olmer pour dresser, en s'appuyant dans un premier temps sur ces données, une carte plus complète de diffusion des amphores romaines en Gaule (Olmer et al., 2013).

10. Il ne faut pas tenir compte de la Corse, qui n'a pas encore fait l'objet d'un dépouillement systématique. type de gisement ou d'objet pour une période donnée : l'interprétation des cartes doit être nuancée par la comparaison avec ce masque.

Une anamorphose ${ }^{11}$, fondée sur le nombre de sites par carré de $50 \mathrm{~km}$ de côté (la surface du carré est augmentée en fonction du nombre de gisements qu'il contient), souligne les contrastes entre ces zones géométriques, peu sensibles aux biais des sources. Elle montre également que le fichier est globalement équilibré, puisque la déformation des bordures de la carte est faible : seul le Nord-Est est un peu plus fourni que les autres régions, tandis que la normandie et les Côtes-d'Armor sont déficitaires (fig. 5) ${ }^{12}$.

\section{Le problème de la chronologie}

Il est nécessaire ensuite d'analyser les données chronologiques. Même pour les non spécialistes, on ne peut plus mettre dans la même enveloppe les princes du premier Âge $\mathrm{du}$ Fer, les paysans et les guerriers de la colonisation celtique, et la société urbanisée des oppida qu'ont décrite César et Posidonios.

La codification par périodes typo-chronologiques admet plusieurs niveaux qui correspondent aux précisions offertes par nos sources. Certains gisements sont seulement attribués à l'Âge du Fer en général (ils ont été ici exclus des tableaux), d'autres globalement aux périodes de Hallstatt ou de La Tène, d'autres à des fourchettes étroites de quelque 70 ans comme le Ha D3 ou LT D2.

La figure 6 (fig. 6) représente les gisements classés par période chronologique en fonction de la date la plus récente de leur occupation. La longueur des rectangles correspond à la durée d'occupation; leur hauteur est proportionnelle au nombre de gisements qui présentent la même fourchette ${ }^{13}$.

11. L'anamorphose en cartographie statistique déforme l'espace $\mathrm{x}, \mathrm{y}$, en fonction d'une valeur particulière. Le logiciel utilisé est Scap Toad. La variable utilisée pour chaque carte est le nombre de sites, il ne s'agit pas d'une variable de densité. L'anamorphose permet de se soustraire de la contrainte de taille de l'espace géographique considérée qui peut exagérer ou minorer la variable à représenter. La vision offerte en minorant, voir en faisant presque disparaitre les territoires avec de faibles valeurs de nombre de sites, renforce leur caractère " périphérique " par rapport à ce qui est étudié. En terme de comparaison (c'est peut-être l'argument le plus valable) elle permet de percevoir plus rapidement les oppositions d'une carte à l'autre.

12. S. Krausz, dans son habilitation soutenue récemment, a utilisé la Base "Patriarche " pour mettre en évidence, du Néolithique à la fin de l'Âge du Fer, les variations significatives des zones de peuplement dans la région Centre à partir des données du Ministère de la Culture. Les résultats sont spectaculaires, même si les descripteurs sont très sommaires.

13. Si en un même lieu il y a une solution de continuité dans l'occupation d'un gisement ou un changement de sa fonction, on fait une autre fiche et on considère qu'il s'agit de deux gisements différents. On retrouve ici la méthode employée par H. J. Eggers et le groupe de la revue Archaeologia Geographica dans les années 1950 à Hambourg (Eggers, 1951). 
Figure 3 : (Voir planche couleur X) Évolution chronologique du centre médian de la répartition des meules. BaseFer mars 2014 ( ). O. Buchsenschutz Figure 3: (See colour plate X) Chronological evolution of the median Center of the distribution of rotary quern-stones.
Figure 4 : (Voir planche couleur XI) Densité des gisements, maillage par carrés de 50 x $50 \mathrm{~km}$ qui rendent mieux compte des concentrations pour des cartes chargées. BaseFer, mars 2014 (C).

Figure 4: (See colour plate XI) Density of deposits, squares of $50 \times 50 \mathrm{~km}$ that better reflect the concentrations for densely populated maps.
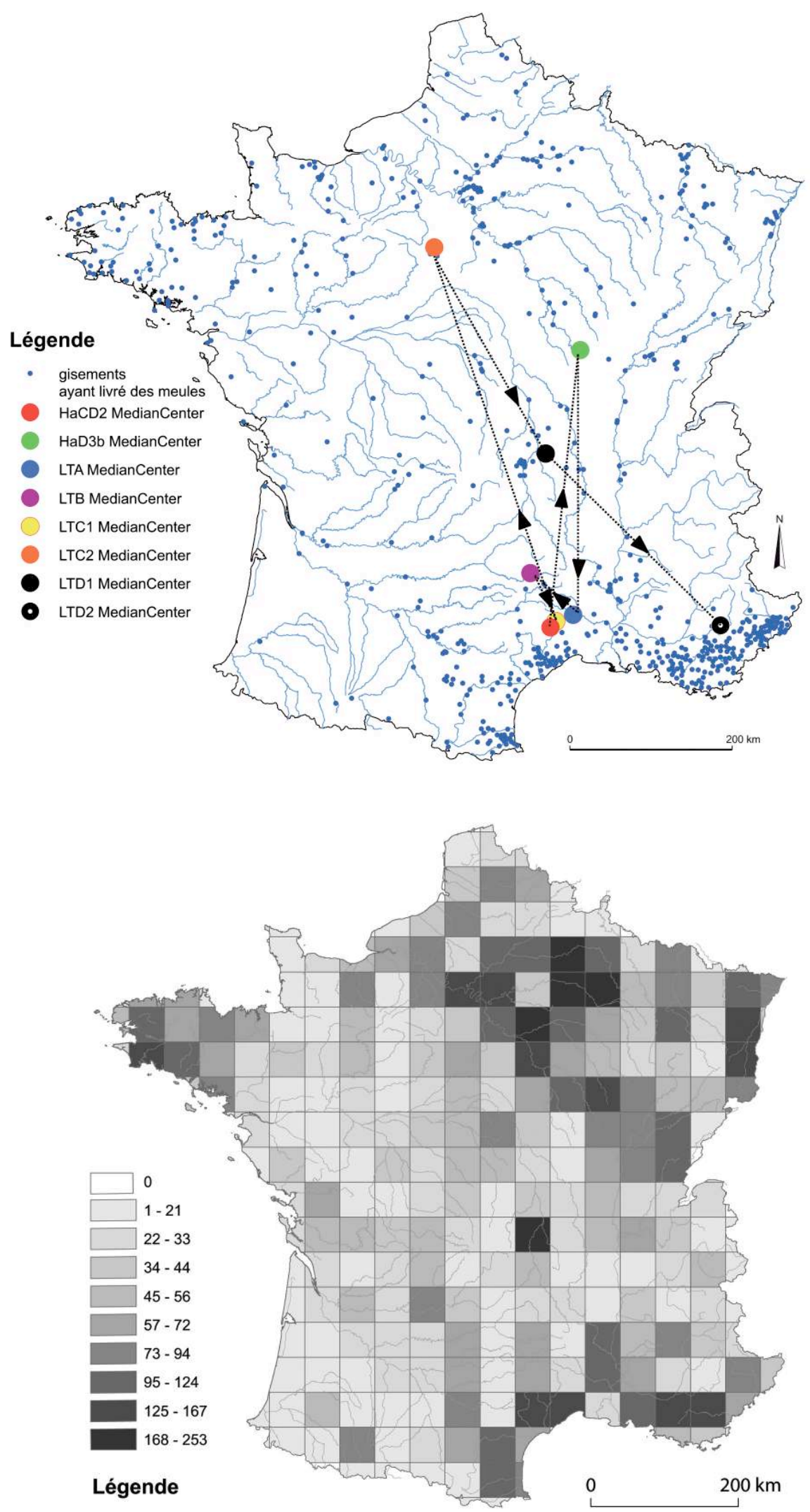

Nombre de gisements par carré de $50 \times 50 \mathrm{~km}$ 


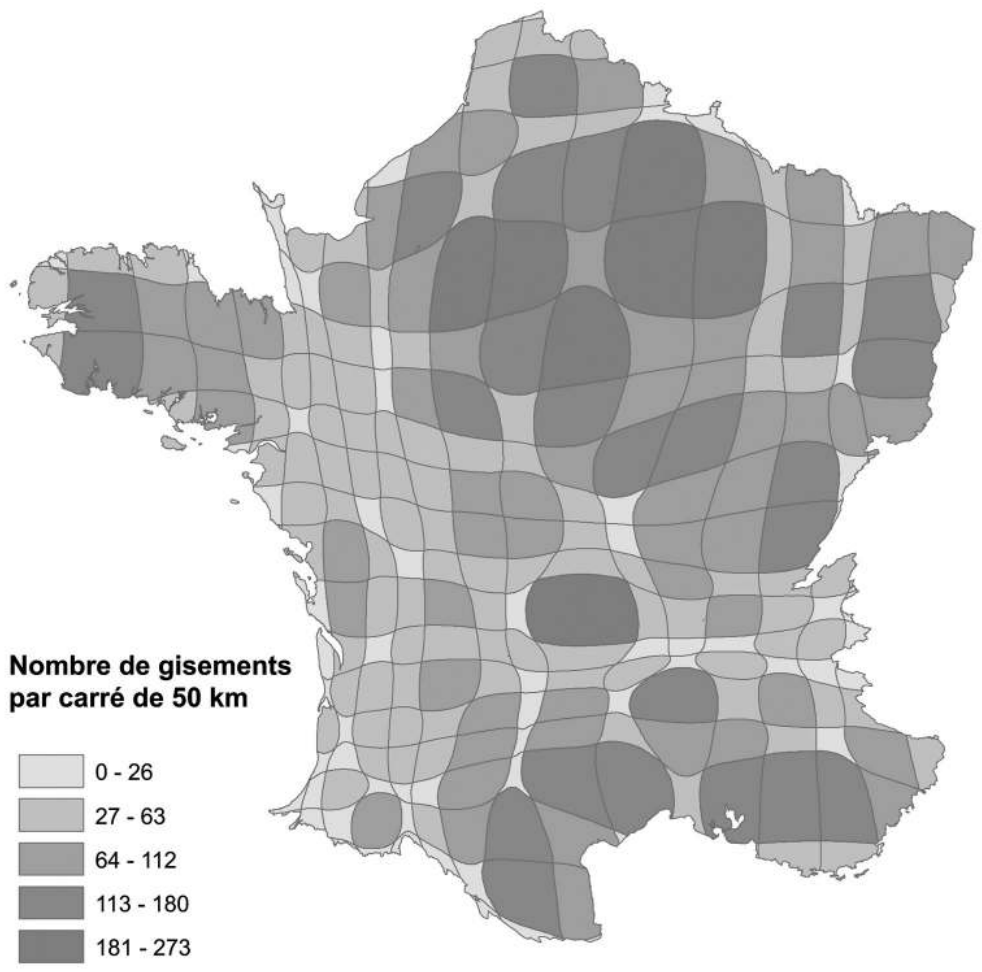

Figure 5 : (Voir planche couleur XI) Anamorphose du groupe 7 (390-345 BC). L'anamorphose prend en compte, pour chaque carré de $50 \mathrm{~km}$ de côté, le nombre de sites qualifiés pour la "structure principale ». BaseFer $\odot$ Ch. Batardy.

Figure 5: (See colour plate XI) Anamorphosis of Group 77 (390-345). Anamorphosis takes into account, for each square of $50 \mathrm{~km}$ of coastline, the number of sites qualified for the "main structure".
Suivant les objectifs que l'on recherche, on sélectionnera les groupes que ce tableau met en évidence : soit les gisements qui entrent dans une fourchette visiblement trop large seront conservés, soit ils seront éliminés du calcul, comme on le fait dans le traitement des nécropoles avec les sépultures mal datées. Il est aussi possible de trier d'abord sur la date d'apparition des sites, ou encore d'exclure les gisements qui sont à la fois mal datés et décrits de façon très vague.

Il est évident à la lecture du tableau obtenu que les fourchettes de datation absolue présentent des seuils liés de fait à la typologie. L'état de la recherche montre qu'on ne peut pas dépasser actuellement la précision du quart de siècle en chronologie absolue.

\section{Une représentation statistique générale des « structures principales"}

Nous avons appelé "structure principale " le premier niveau d'analyse des gisements : habitat, ensemble funéraire, dépôt, sanctuaire, atelier. Le croisement des données statistiques de base, structure principale sur un axe, chronotypologie sur l'autre, donne une vision schématique de l'état actuel des connaissances sur l'Âge du Fer en France (fig. 7).

Nous avons sélectionné les gisements qui n'étaient occupés que pendant une période typologique précise, par exemple
Ha D2, LT C1 etc. À l'issue de ce tri, il en restait 1706. Nous avons conservé douze périodes, les plus anciennes n'étant pas détaillées parce qu'elles réclament encore un examen plus approfondi. Nous n'avons pas non plus retenu dans cette base les raffinements des derniers siècles (LT D 1a, LT D1 b etc.), qui varient encore beaucoup d'une région à l'autre.

Les ateliers sont attestés à plusieurs périodes; on notera surtout les vides du Ha D2 à LT A, qui doit être sans doute révisé avec les découvertes récentes, et le vide de LT B2, qui semble mieux correspondre aux connaissances actuelles.

Les dépôts, très abondants dans les phases anciennes, décroissent rapidement au $\mathrm{Ha}$ D2 pour remonter un peu au Ha D3 et à LT A. Pour les périodes les plus récentes, nous n'avons pas comptabilisé les monnaies, même si les trésors, très nombreux mais souvent mal datés dans les publications qui ont été dépouillées ${ }^{14}$, se substituent à la pratique ancienne.

Les sites funéraires semblent bien prendre au Hallstatt la place que les dépôts occupaient aux phases précédentes. Ils restent très nombreux jusqu’à $\mathrm{LT} B$, comme on le vérifie ici.

14. La datation des séries monétaires gauloises a énormément progressé ces dernières années mais les recensions bibliographiques que sont les CAG véhiculent un état de la recherche fortement dépassé dans ce domaine. 


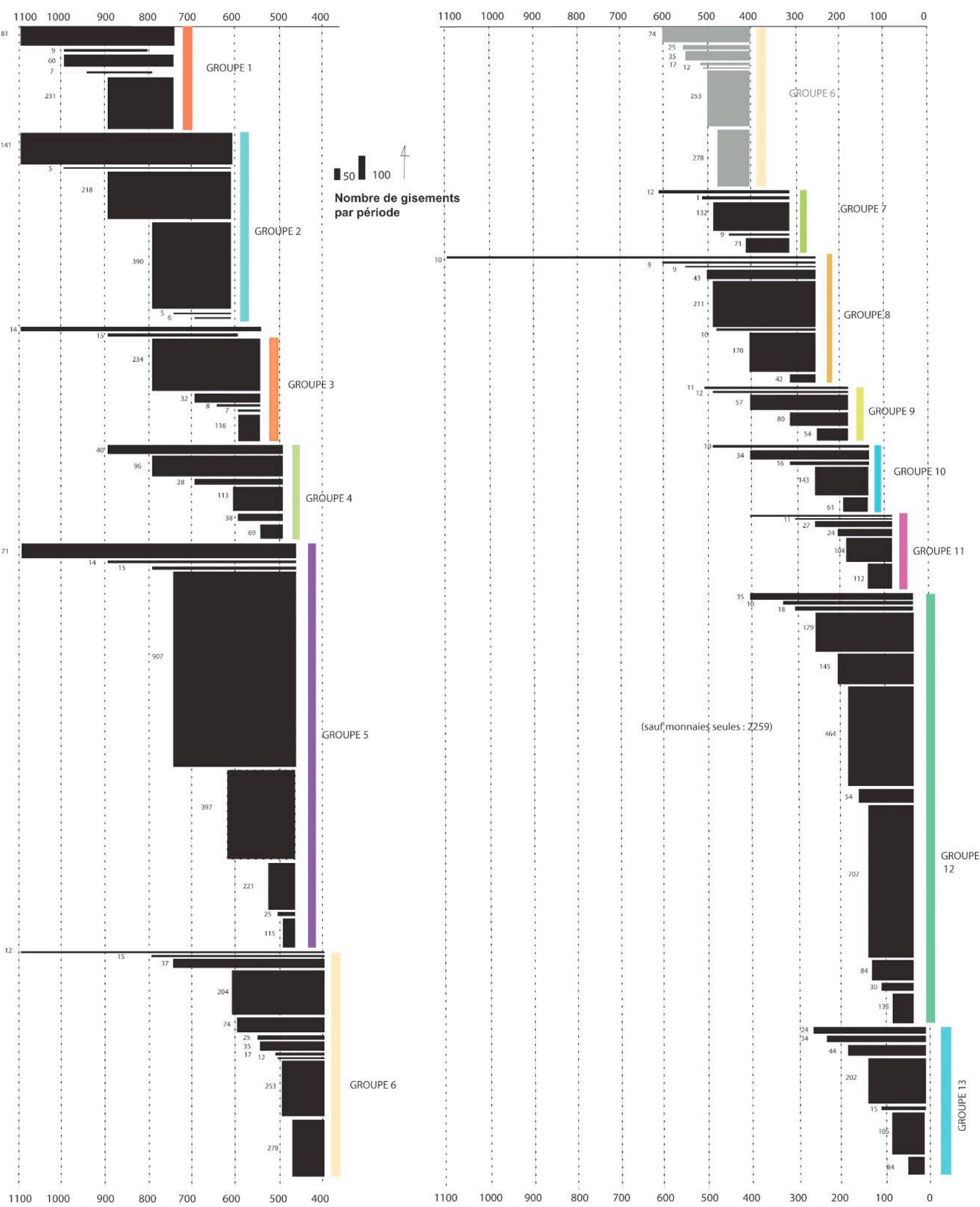

Figure 6: (Voir planche couleur XII) Nombre de gisements classés par phase typo-chronologique. Basefer mars 2014 ( Figure 6: (See colour plate XII) Number of settlements classified by typo-chronological phase. 


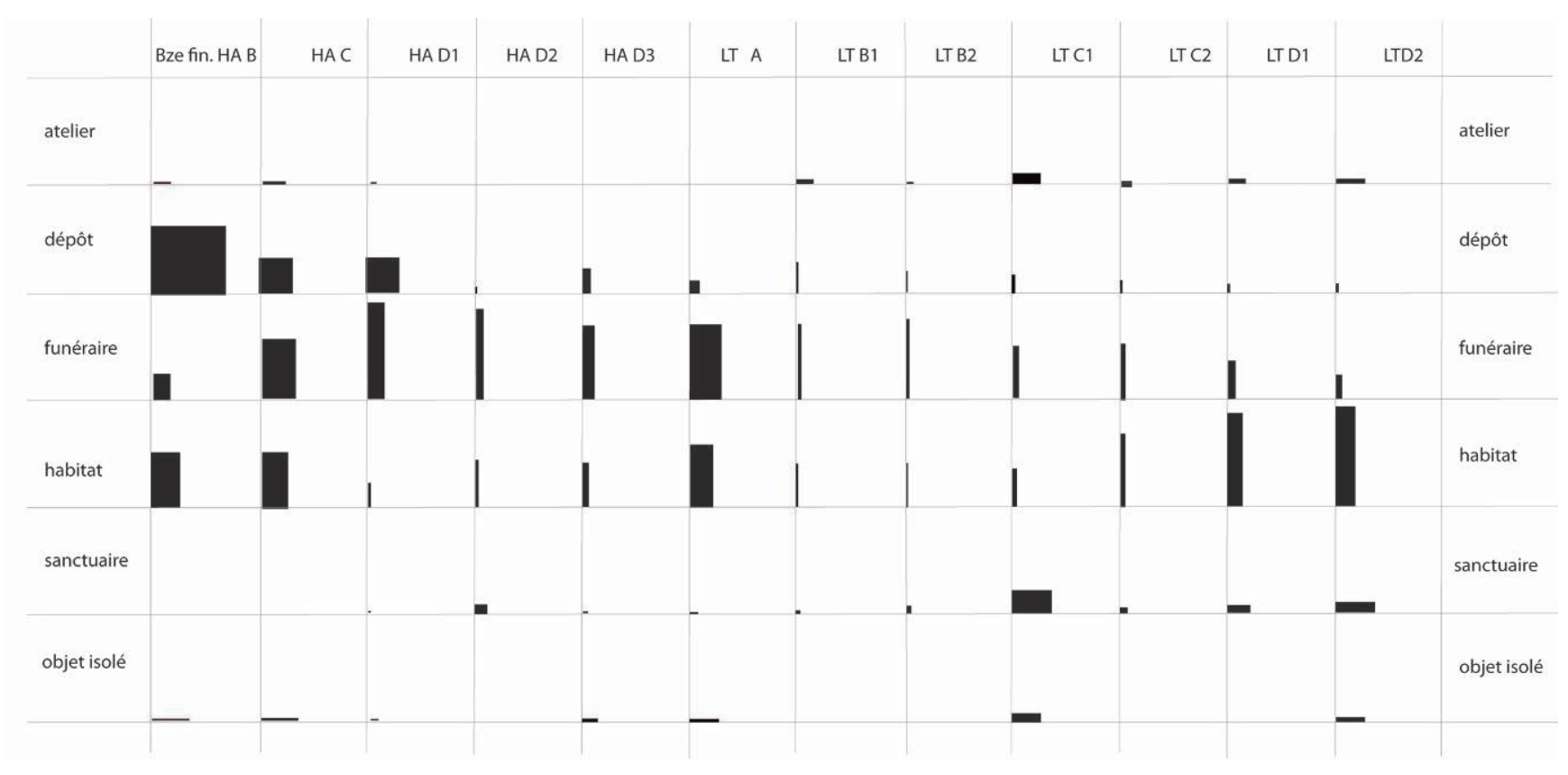

Figure 7 : Représentation en pourcentages croisés des «structures principales " par période.

Figure 7: Representation in cross percentages "principal structures" by period.

Leur proportion est particulièrement importante au $\mathrm{Ha} \mathrm{C}$ et D1 et à LT A.

Les grandes phases de l'habitat sont présentes aux périodes Ha B et C, LT A, et LT D. En proportion par rapport aux autres structures principales, ils sont largement dominants à LT C2 et D. Si on y ajoutait les innombrables fermes fouillées en sauvetage, dont seulement une faible proportion est publiée, cette augmentation serait encore plus importante.

Les sanctuaires, bien connus pour LT C1, ne se limitent pas à cette période : ils sont présents dès le $\mathrm{Ha} \mathrm{D} 2$, à travers les concentrations de stèles notamment, et perdurent jusque pendant la romanisation.

Ce tableau donne une idée de la richesse et des limites de nos informations. Un peu plus d'un dixième des gisements de la base sont classés dans des cases chronologiques bien définies. Le déséquilibre entre sites funéraires et sites d'habitat est généralement résorbé, ce qui permettra de mieux mettre en rapport ces deux éléments fondamentaux pour définir un peuplement. Les autres catégories, ateliers, sanctuaires, sont relativement bien attestées à plusieurs périodes. Enfin les dépôts jouent un rôle plus important que prévu, surtout si on considère que les dépôts monétaires s'inscrivent dans la continuité des anciennes traditions.

\section{Analyses globales}

Un sondage rapide dans la bibliographie, et notamment dans les Cartes Archéologiques de Gaule, explique certaines différences. Les plus fortes inégalités, - fortes densités pour le Var, densités très faibles pour la Manche et le Gers -, s'expliquent ici par des séries particulières, les petites enceintes de hauteur, là par la légèreté de l'enquête, soulignée par les auteurs eux-mêmes. Malgré cette difficulté incontournable, des analyses plus détaillées de l'ensemble de l'hexagone sont significatives.

\section{Anamorphoses (fig. 8)}

Si l'intensité des recherches ou la nature des terrains expliquaient ces inégalités, les différences entre les départements seraient pratiquement les mêmes d'une période à l'autre. Or on peut constater que ce n'est pas le cas (fig. 8). Le phénomène qui apparaît là est véritablement " historique ". Les variations chronologiques sont très fortes par exemple pour les départements du Nord, de l'Hérault, du Finistère. Le département de la Seine-et-Marne, qui bénéficie d'une publication très récente intégrant d'importantes fouilles de sauvetage, varie beaucoup du groupe chronologique 1 au groupe 13 (cf. ci-dessus « Le problème de la chronologie »), alors que les conditions de vie comme les possibilités de détection des sites n'ont pas changé pendant tout l'Âge du Fer. Des variations sont également clairement perceptibles en Alsace, non seulement par rapport à ce qu'on appelle là-bas la France de l'intérieur, mais aussi entre le Haut- et le Bas-Rhin dont les spécialistes soulignent les différences à l'Âge du Fer. 

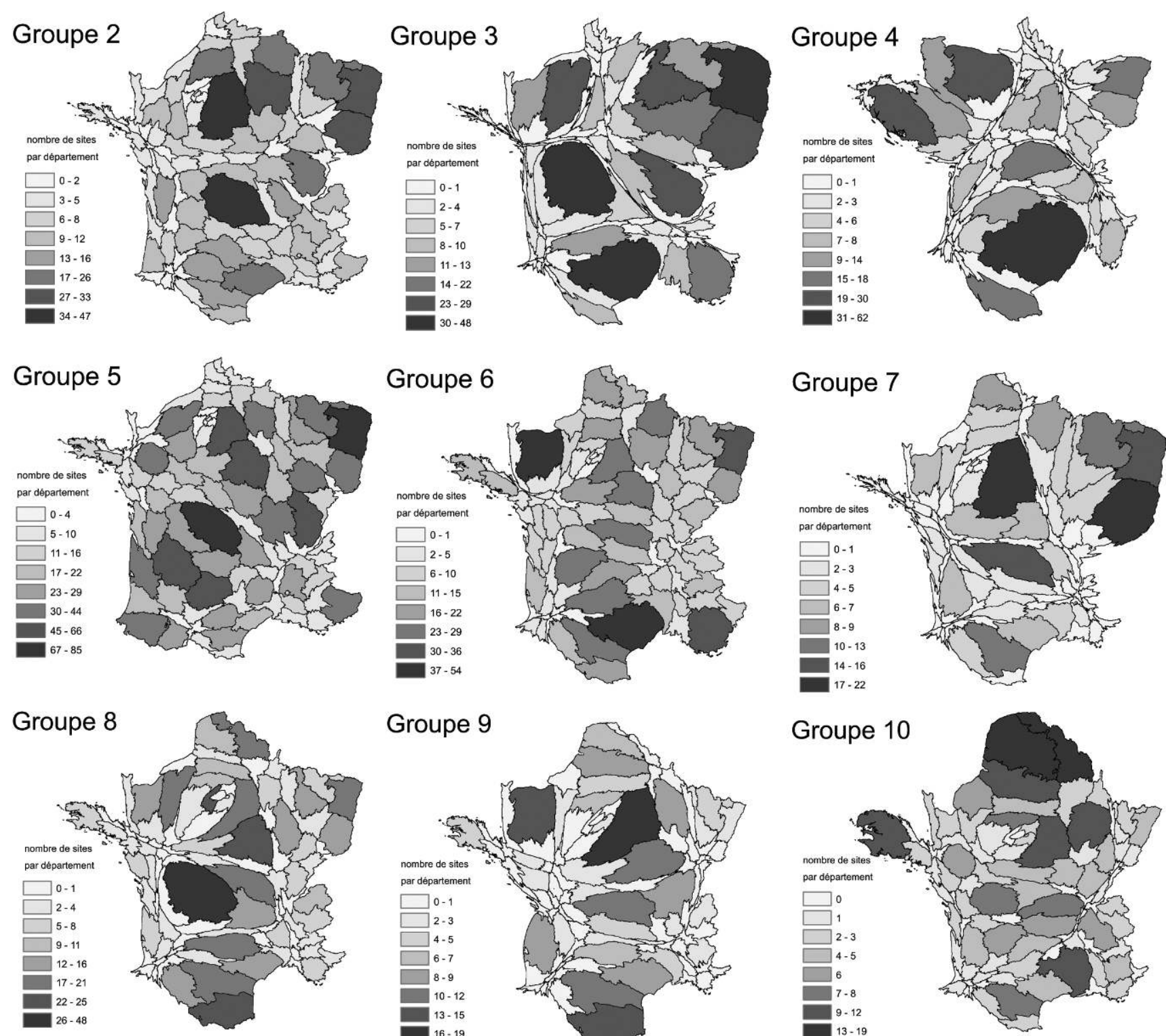

Groupe 9
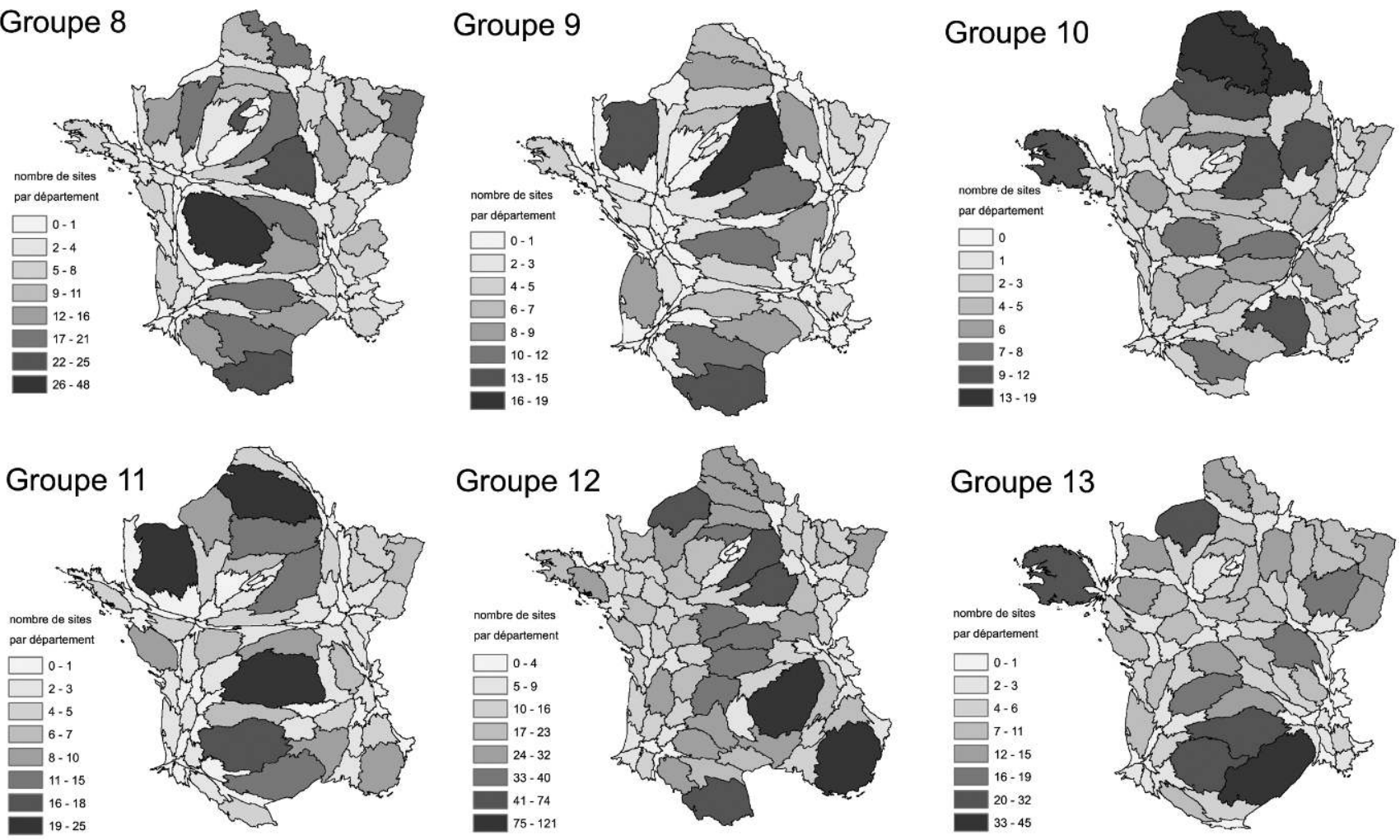

BaseFer mars 2014 ㄷ E. Hiriart

Figure 8 : (Voir planche couleur XIII) Anamorphoses du nombre de sites par département et par période (12 groupes chronologiques) BaseFer mars 2014 (), E. Hiriart.

Figure 8: (See colour plate XIII) Anamorphosis of the number of settlements per Department and per period (12 chronological groups) 
Par rapport à une image de référence de répartition homogène qui serait une France non déformée dans notre cas, on peut observer des cartes qui s'écartent beaucoup d'une répartition homogène, comme le groupe 4 de la figure 8 , par rapport à une carte qui n'en s'en écarte pas ou très peu, comme c'est le cas pour le groupe 12.

Il ne faut pas oublier en analysant ces cartes que la trame départementale ne rend évidemment pas compte des inégalités internes : la plupart des sites du département du Puyde-Dôme, comme on l'a vu sur d'autres cartes, sont en fait regroupés dans la Limagne.

\section{Analyse de la Base par Classification Ascendante Hiérarchique (fig. 9)}

Nous avons testé une autre méthode pour rendre compte de l'évolution régionale et chronologique des «structures principales ", tels qu'ils ont été perçus dans les recherches archéologiques. Ce bilan très global ne représente bien sûr qu'un survol de la réalité historique; il peut toutefois orienter les recherches futures en révélant quelle visibilité ont ces différents types de sites suivant les périodes et les régions (fig. 9).

Ici le découpage départemental est abandonné au profit d'une maille de 50 x $50 \mathrm{~km}$ qui représente une base statistique suffisante pour établir des corrélations entre les différents types de sites ${ }^{15}$.

Nous ne proposons pas ici une interprétation historique. Les questions que pose cette analyse statistique mériteraient d'être discutées par un groupe de chercheurs représentant les différentes régions, périodes, et spécialités. Nous nous contentons donc ici d'attirer l'attention des lecteurs sur les concentrations, les absences, les densités que les cartes révèlent, en espérant réunir dans un avenir proche cet aréopage idéal pour progresser. La représentation sur un même document de l'évolution dans le nord et dans le midi de la France mériterait à elle seule une discussion approfondie.

Les tableaux dessinés au-dessus de chaque carte représentent les résultats de la classification. Nous avons retenus 5 classes, parce qu'au-delà l'interprétation devient plus nuancée, et donc plus difficile à lire. Chaque classe est caractérisée par la proportion des "structures principales " (habitat, funéraire etc.) les unes par rapport aux autres. Le graphique représente la différence à la moyenne, positive ou négative, de chaque catégorie dans la classe. Nous avons simplifié l'interprétation en retenant seulement la dominante, - habitat, ou funéraire etc. -, mais on pourrait faire une lecture plus subtile en tenant compte des catégories déficitaires, ou bien

15. Nous avons utilisé le programme Philcarto @ C de Philippe Waniez qui permet de dresser une cartographie à partir des résultats statistiques. de la vigueur de la catégorie dominante, exprimée par la longueur du trait en écart-type.

Plusieurs fois deux classes sont dominées par les dépôts, ou par les habitats. Nous avons exprimé par deux couleurs différentes les carrés qui leur correspondent, mais en restant dans la même gamme (le rose ou le beige pour les dépôts, le vert pour l'habitat, le rouge pour le "funéraire", le bleu pour les sanctuaires, le jaune pour les « ateliers »). La même gamme a été utilisée pour toutes les cartes afin de permettre une lecture comparée des différentes périodes. Les 13 périodes sont celles qui ont été obtenues par le calcul présenté dans la figure 6 .

- Carte du groupe 1 : Les zones dominées par les dépôts sont nombreuses, surtout dans l'Ouest.

- Carte du groupe 2: Au Hallstatt C ( $\mathrm{Ha} \mathrm{C})$ les zones caractérisées par une majorité de structures funéraires ont dépassé les dépôts. Elles dessinent une écharpe du nordest au sud-ouest du territoire, qui était déjà visible dans les anciennes cartes. Les ateliers sont plus nombreux et les premiers sanctuaires apparaissent.

- Carte du groupe 3 : La période du Ha D1 est particulièrement pauvre en gisements. Les habitats l'emportent largement, tandis que les ateliers atteignent le même score que les structures funéraires.

- Carte du groupe 4 : À la période du Ha D2, les régions sont plus contrastées : les dépôts dominent la Bretagne, les habitats et les gisements funéraires se partagent le reste du territoire, les sanctuaires réapparaissent alors que les ateliers ont momentanément disparu.

- Carte du groupe 5 : Ils reviennent en force au Ha D3, période très riche malgré sa courte durée, mais les habitats et les sites funéraires dominent largement. On remarque aussi l'auréole de dépôts qui subsiste dans les marges orientales de la Bretagne.

- Carte du groupe 6 : La densité est moins forte, mais toutefois encore importante à La Tène A (LT A), où les sites funéraires dominent largement, sauf en Provence et dans le Nord.

- Carte du groupe 7 : Le nombre de gisements est beaucoup plus faible à LT B1 et les secteurs marqués par une dominante sont restreints au quart Nord-Est (funéraire) et à la région du delta du Rhône (habitat).

- Carte du groupe 8 : La période suivante réunit le double de gisements, les ensembles funéraires dominent encore plus le Nord-Est, tandis que les secteurs où l'habitat est le premier sont très dispersés.

- Carte du groupe 9 : On revient à un score encore plus faible que celui de LT B1 pour LT C1; les secteurs à dominante funéraire ont glissé vers le centre du Bassin parisien, 
tandis que l'habitat, toujours dispersé, s'impose dans le midi méditerranéen.

- Carte du groupe 10 : LT C2 reprend dans ses grandes lignes la distribution précédente, mais l'habitat et aussi, ce qui est plus surprenant, les gisements funéraires, dessinent une mosaïque contrastée dans le centre du Bassin parisien. Il faut relever également la présence des sanctuaires et des ateliers.

- Carte du groupe 11 : L'habitat l'emporte largement sur le funéraire à LT D1. Les secteurs dominés par les sanctuaires sont très nombreux.

- Carte du groupe 12: La carte de LT D2 offre une large part aux dépôts car, dans l'état actuel des données les trésors et dépôts monétaires, qui sont extraordinairement nombreux, sont attribués à cette période, et n'ont pas été distingués des monnaies isolées. Les gisements funéraires ne résistent pas à cette invasion, ateliers et habitats demeurent.

- Carte du groupe 13 : Nous avons conservé une période pour les débuts de la romanisation (27-1), qui souligne le maintien des habitats, des ateliers, et une progression des sanctuaires, peut-être seulement parce que leur "pétrification " permet de les repérer plus facilement.

Pour mesurer l'extension spatiale et chronologique de phénomènes généraux, comme le développement des tertres funéraires, la proportion entre les rites d'incinération ou d'inhumation, le port des colliers, bracelets et fibules, l'échelle et le degré de précision que nous avons choisis semble raisonnable. Bien sûr nous sommes impatients d'aller plus loin dans le détail, mais il y a des spécialistes pour cela : ils pourront ajouter à leur fichier la description des gisements et le mobilier qui accompagne leur objet de recherche préféré.

\section{UNE ÉTUDE DE CAS : LES MONNAIES}

Nous avons choisi de présenter l'exemple des monnaies qui constituent un bon traceur de la pénétration d'un nouvel outil d'échange sur ce territoire. La monnaie, inventée par les Grecs, ne fait son apparition en Gaule qu'au III $^{\mathrm{e}}$ s. av. J.-C. Auparavant, l'unité de valeur est l'or, stocké sous forme de bijoux. Les colonies grecques d'Ibérie et de Gaule n'ont jamais frappé que de l'argent et du bronze. Les régions gauloises adoptent la monnaie avec un certain décalage selon leur relation avec les peuples méditerranéens et leur besoin en numéraire. Ainsi, alors que les trésors sont nombreux dans l'Ouest armoricain, les monnaies y restent rares dans l'habitat. (Gruel et al., 1990; Gruel et Pion, 2009) Les cartes commentées ici correspondent aux communes sur lesquelles est mentionnée la présence de monnaie(s) sur les sites. Si on examine globalement la circulation, en Gaule, des monnaies gauloises, des monnaies romaines, des monnaies de Marseille et des autres monnaies grecques, on constate que les données saisies pour les monnaies gauloises rendent compte de leur circulation avec un décentrement attendu à hauteur de la boucle de la Loire, comme le montre l'ellipsoïde de déviation (fig. 10a). Les émissions massaliotes se diffusent à partir de Marseille sur les agglomérations (fig. 10, b, points bleu), mais restent fortement concentrées dans la basse vallée du Rhône (fig. 10e). L'anamorphose de leur répartition par département caricature ce poids de la Gaule méditerranéenne, et dessine bien l'image de la Gaule vue de Marseille (fig. 10c). Cette carte déformée par une diffusion monétaire exclusivement concentrée sur les départements du Sud, redresse les Pyrénées et semble déformer les fleuves qui, comme dans le texte de Strabon, semblent orientés nordsud.

La diffusion des frappes de Marseille numéraire (fig. 10b et e) semble complémentaire de celle des monnaies romaines, comme le montrent les cartes de densité des sites qui ont livré du numéraire. L'outil Densité répartit une quantité mesurée d'une couche de points en entrée sur l'ensemble d'une carte, de manière à générer une surface continue. Dans chaque commune, un nombre donné de sites ont fourni des monnaies. Cette valeur par commune induit une densité d'usage monétaire sur l'ensemble de la surface.

Les pièces romaines qui, d'après les contextes archéologiques, circulent essentiellement à La Tène D2, se concentrent sur certains sites de la côte occidentale de la Méditerranée, probablement en lien avec la province romaine d'Espagne et la création de Narbonne, et d'autre part sur des gisements situés le long des grands fleuves (fig. 10d). Les découvertes des autres monnaies grecques suivent un schéma différent avec une forte concentration en Catalogne, au contact des comptoirs grecs d'Espagne, le long de la Garonne puis de la côte atlantique. Un autre ensemble, qui correspond à la diffusion des statères de Philippe de Macédoine, se dessine dans la moyenne et la haute vallée du Rhône ainsi que dans la basse vallée de la Loire, originaire soit de la voie du Danube et du Rhône, soit de Marseille (fig. 10c, points rouges et fig. 10f).

L'examen des alliages utilisés pour les monnaies gauloises est révélateur de traditions culturelles différentes plus ou moins influencées par le monde méditerranéen. Nous obtenons trois cartes très différentes pour l'or (fig. 11a), l'argent (fig. 11b) et le bronze (fig. 11c) et nous avons ensuite testé plusieurs méthodes pour mettre en évidence des dominantes par carte de densité (fig. 11d, e, f) et par anamorphose (fig. 12a, b et c). La carte de points dilue visuellement l'information; la carte de densité par site 

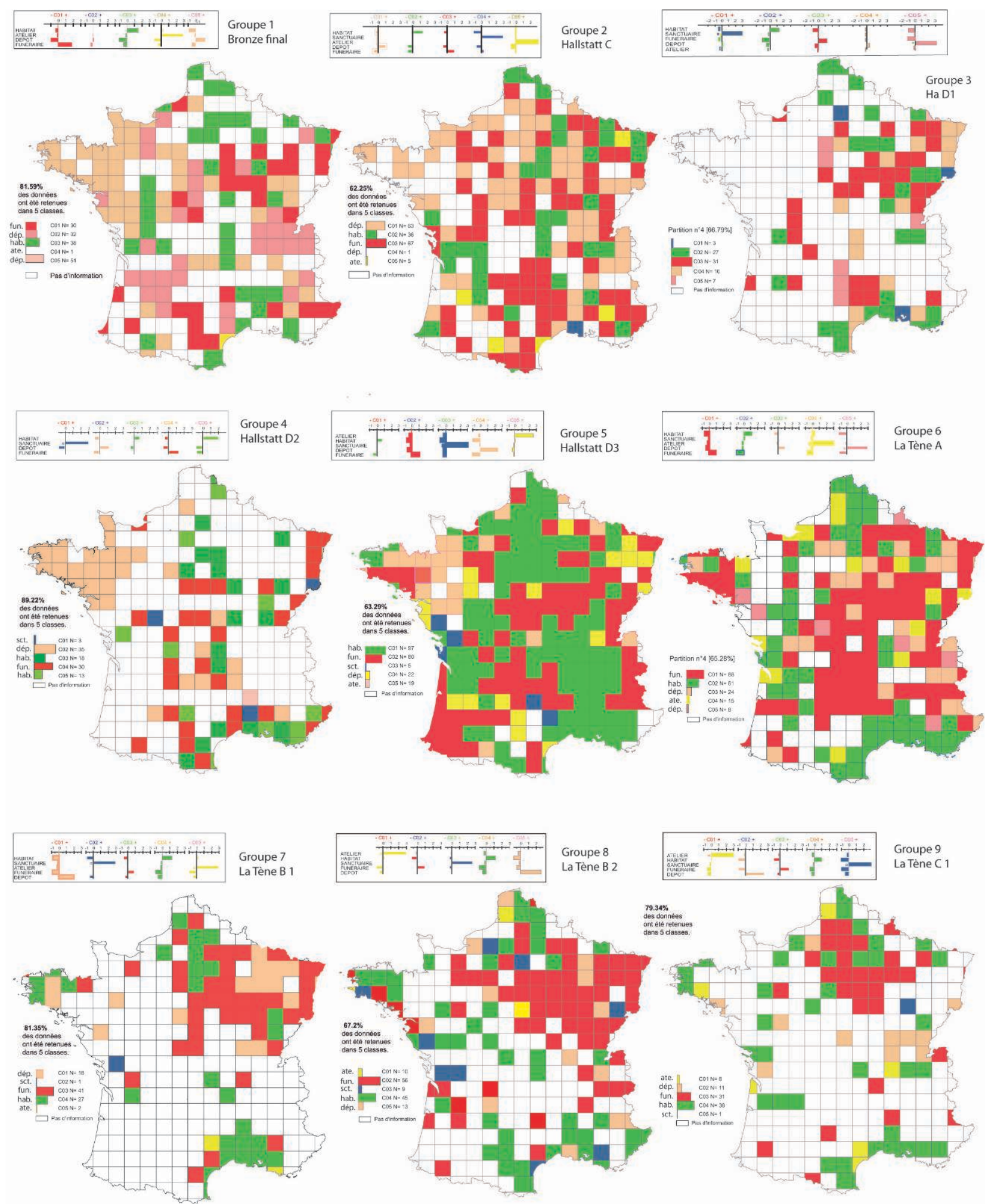

Fait avec Philcarto * 06/10/2014 Ch. Batardy

Figure 9 : (Voir planches couleur XIV-XV) Classification Ascendante Hiérarchique sur une grille de $50 \mathrm{~km}$ x $50 \mathrm{~km}$ des « structures principales »: Ch. Batardy DAO, O. Buchsenschutz, données de la Basefer mars 2014 (C), Philcarto (.

Figure 9: (See colour plates XIV-XV) Agglomerative Hierarchical Clustering on a grid of $50 \mathrm{~km} \times 50 \mathrm{~km}$ of the "principal structures". 

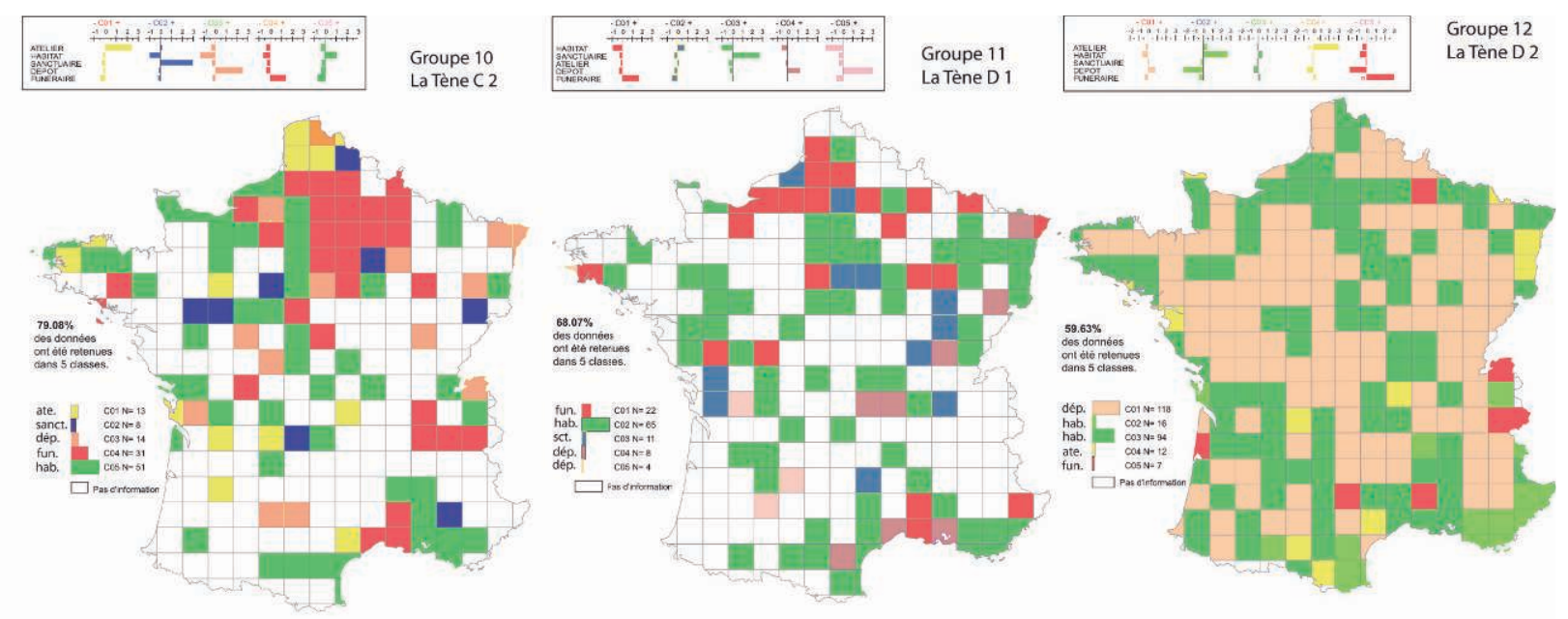

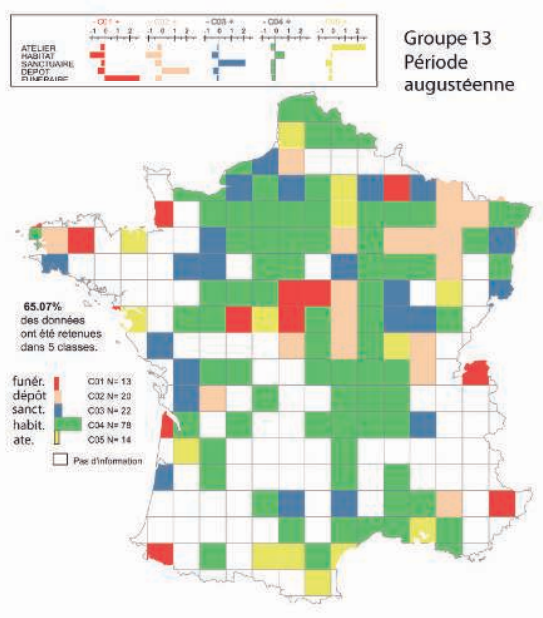

Fait avec Philcarto 06/10/2014 Ch. Batardy

lisse ici les données à un niveau de compréhension régionale (fig. 11). L'anamorphose par département (fig. 12) caricature les résultats en faisant quasiment disparaître les régions faiblement représentées. On voit une concentration nette de l'or monnayé sur la Gaule chevelue avec un poids particulier de la Gaule Belgique et de l'Armorique. Les monnaies d'argent incluant les monnaies de Marseille et les monnaies grecques se concentrent sur le littoral méditerranéen, et remontent par la vallée du Rhône et de la Saône (zone du denier gaulois) et par la vallée de la Garonne (domaine des monnaies à la croix). Les cartes des bronzes recouvrent plusieurs réalités, aux chronologies et répartitions différentes avec la masse des potins tout d'abord dans un quart nord-est de la Gaule, englobant le Centre-Est et la Gaule Belgique, auxquels se superposent les bronzes frappés de La Tène $\mathrm{D}$, tandis que sur le littoral méditerranéen on trouve plutôt des bronzes épigraphes de Narbonnaise et surtout des bronzes de Marseille ou leurs imitations. Sur toutes les cartes se dégagent les grands sites bien étudiés
Figure 9 (suite)

Figure 9 (following)

comme Bibracte, Orléans, Clermont-Ferrand, Lattes, VieilToulouse, Marseille... Les données dépouillées provenant en partie des CAG ne peuvent être exploitées pour la chronologie, les datations proposées lors de leur publication ont maintenant été entièrement revues et précisées pour la plupart des séries monétaires.

\section{CONCLUSION}

Le but de cet article est de présenter les nombreuses possibilités de l'analyse statistique ou spatiale sur un corpus dont nous pensons avoir bien mis en évidence qu'il est aujourd'hui équilibré sur le territoire métropolitain. Nous avons montré que les fourchettes chronologiques sont encore pour l'Âge du Fer fortement liées à la typo-chronologie. Il est trop tôt pour l'abandonner, mais les résultats que nous avons obtenus montrent qu'on peut travailler à améliorer cette situation. Les différences de densité ou de proportion des catégories de gisements par période reflètent des évolutions à valeur historique, les biais dus à l'état des recherches sont faibles à cette échelle et facilement mesurables.

Nous avons surtout dans nos commentaires cherché à attirer l'attention du lecteur sur les différences régionales ou chronologiques sans prétendre expliquer la nature du phénomène, qui demande le concours des spécialistes de la période.

Cette analyse préliminaire ouvre de nombreuses pistes de recherches nouvelles pour lesquelles nous pouvons voir en quelques clics si la documentation permet de proposer un 


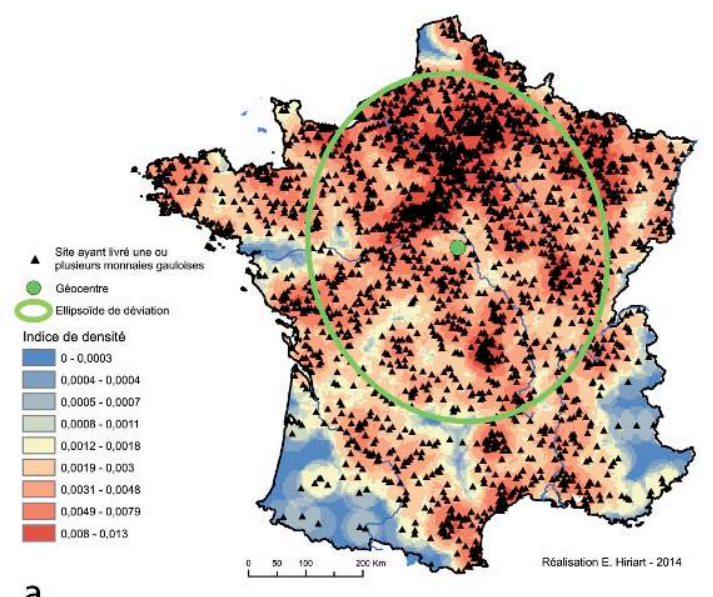

a

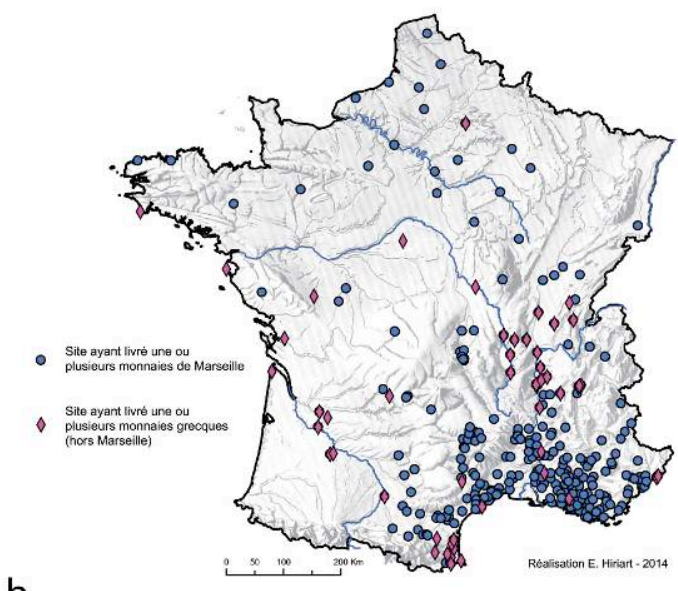

$\mathrm{b}$

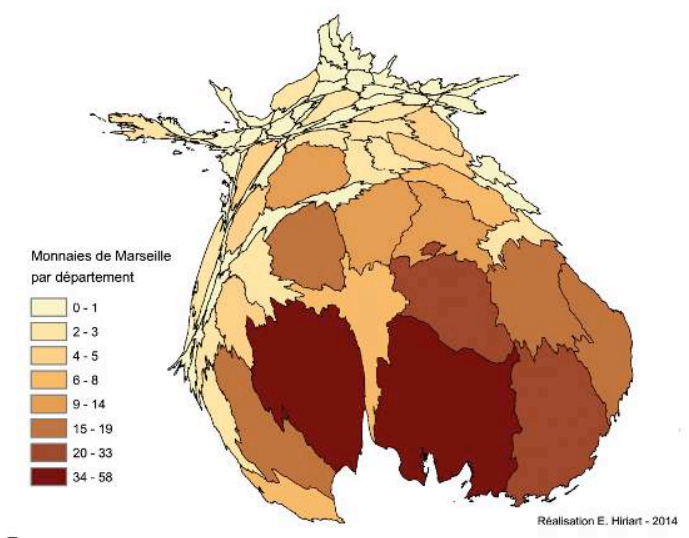

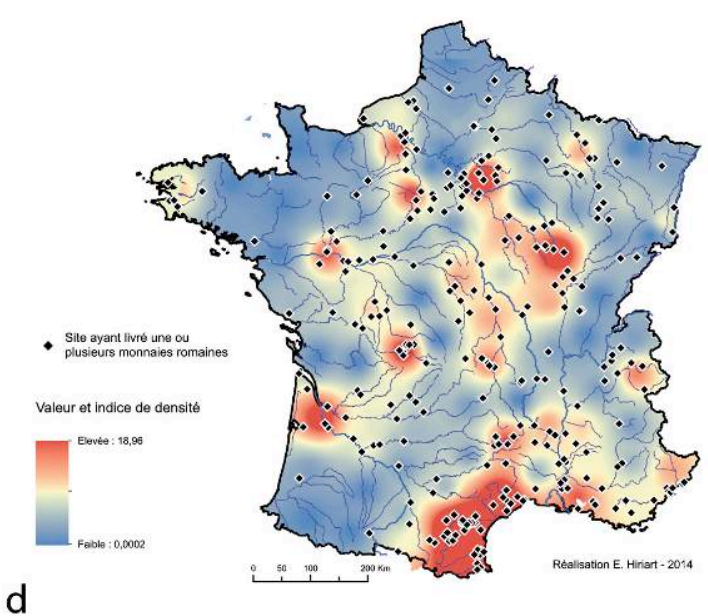

d

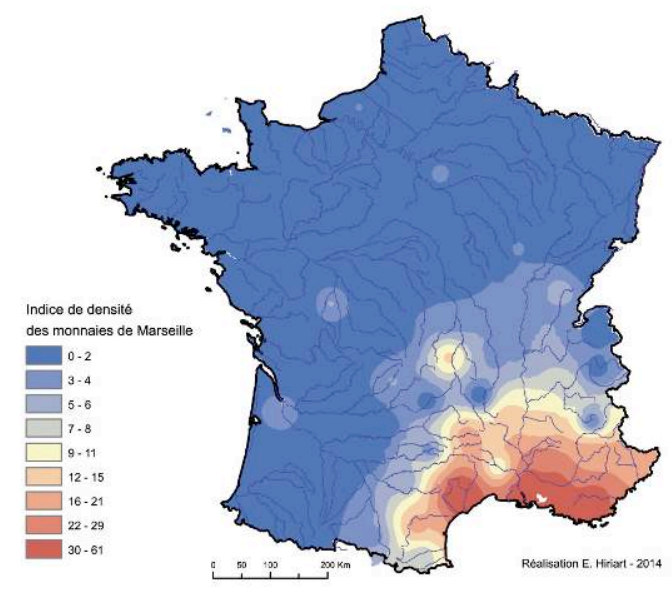

e

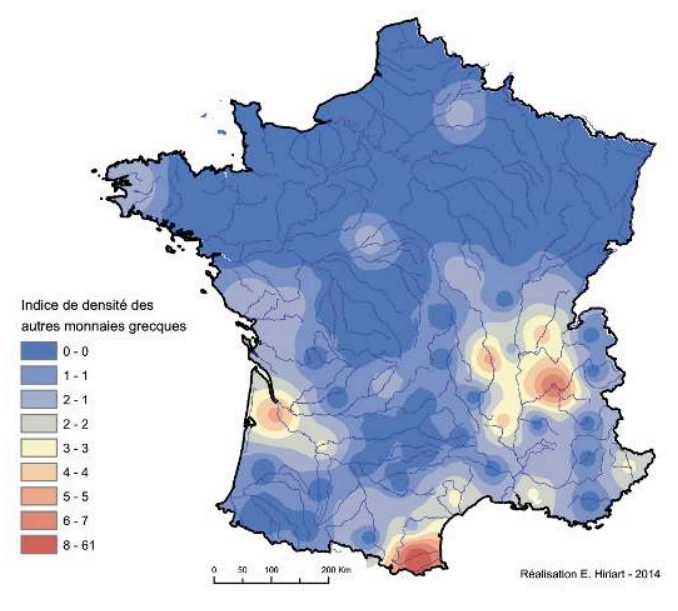

Figure 10 : (Voir planche couleur XVI) Diffusion des monnaies en Gaule, III ${ }^{\mathrm{e}}$ s.- $\mathrm{I}^{\mathrm{er}}$ s. av. J.-C. : a) Les monnayages gaulois, b) Les gisements avec des monnaies de Marseille (bleu) et d'autres monnaies grecques (rouge), c) Anamorphose sur la diffusion des monnaies de Marseille d) Les gisements avec monnaies romaines et la densité des sites, e) La densité des gisements avec des monnaies de Marseille, f) La densité des gisements avec d'autres monnaies grecques (Philippe de Macédoine et Comptoirs ibériques) K. Gruel, DAO, E. Hiriart. BaseFer mars 2014 (C)

Figure 10: (See colour plate XVI) Coins dissemination in Gaul, 3rd-1st c. BC: a) the Gallic coins, b) deposits with Massalia's coins (blue) and other Greeks'coins (red) c) anamorphosis by Department of the presence of Massalia's coins, d) deposits with Roman coins and density of sites, e) density of deposits with Massalia's coins, $f$ ) density of deposits with other Greek coins (Philip of Macedonia and Greeks cities of Iberia). 


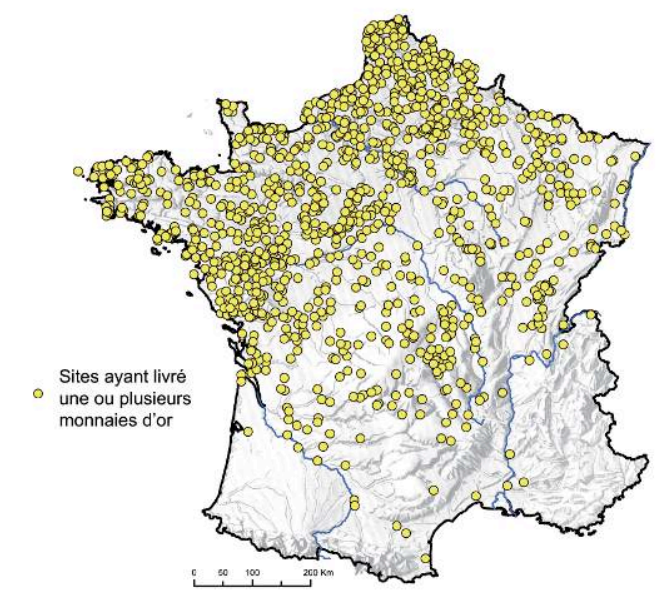

a

b
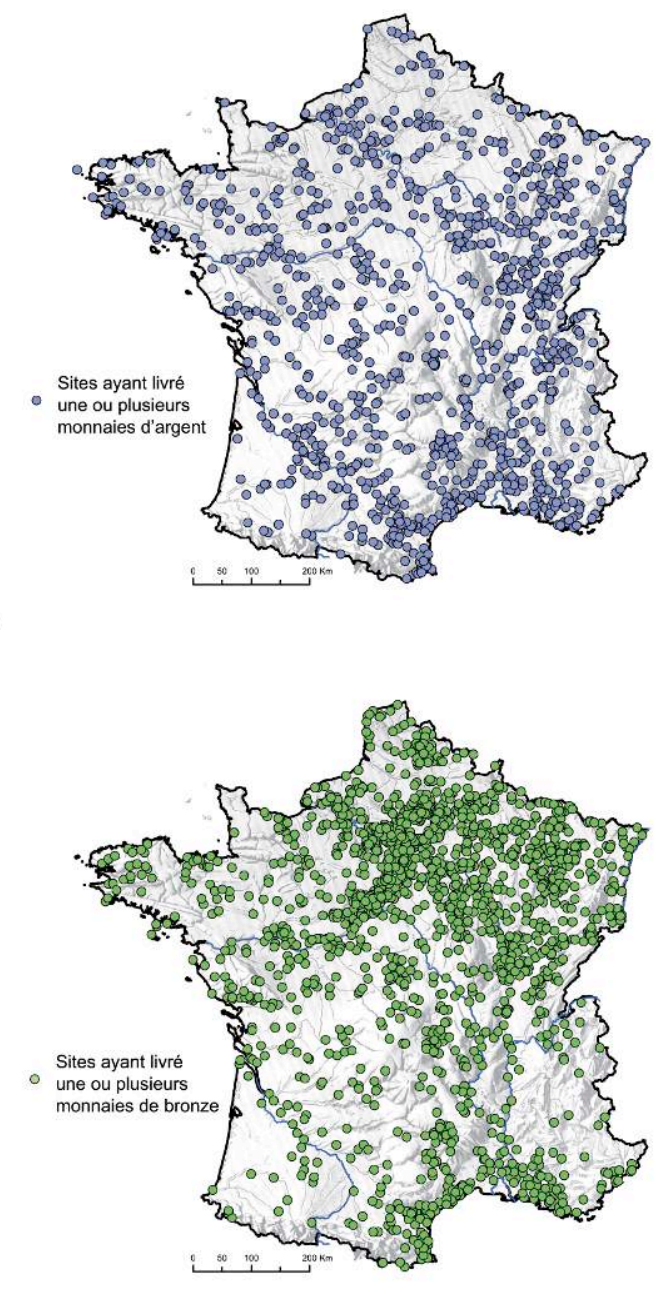
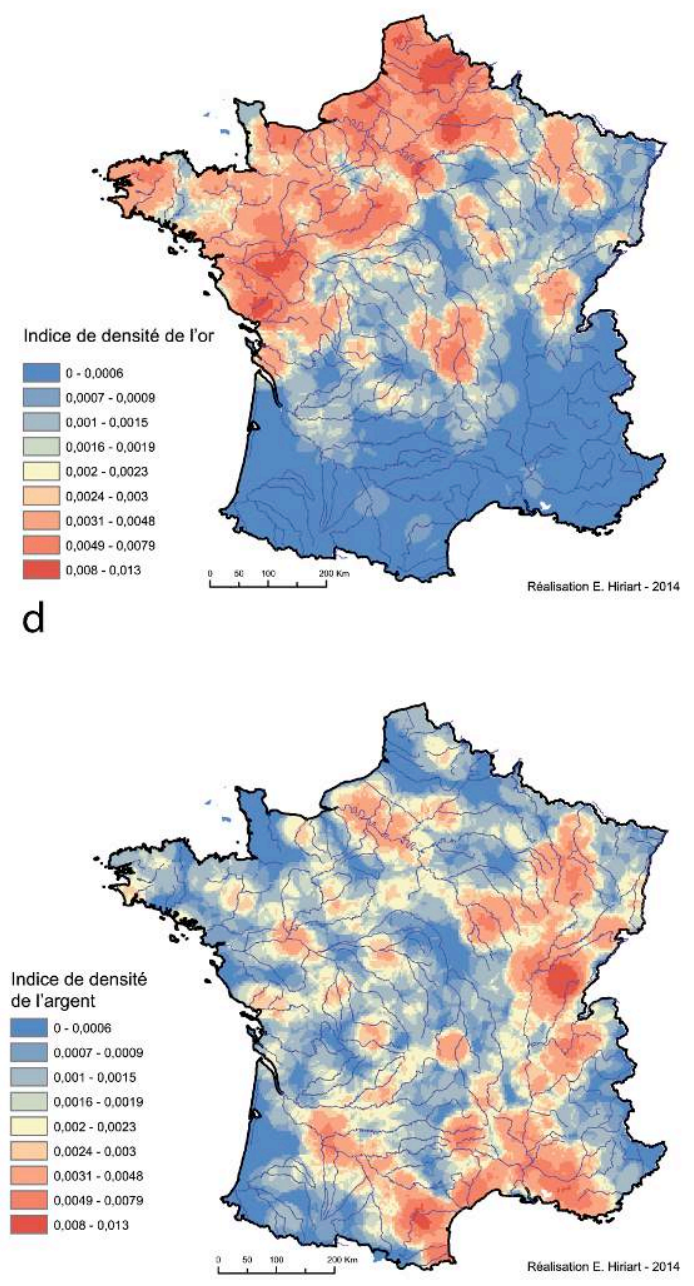

e

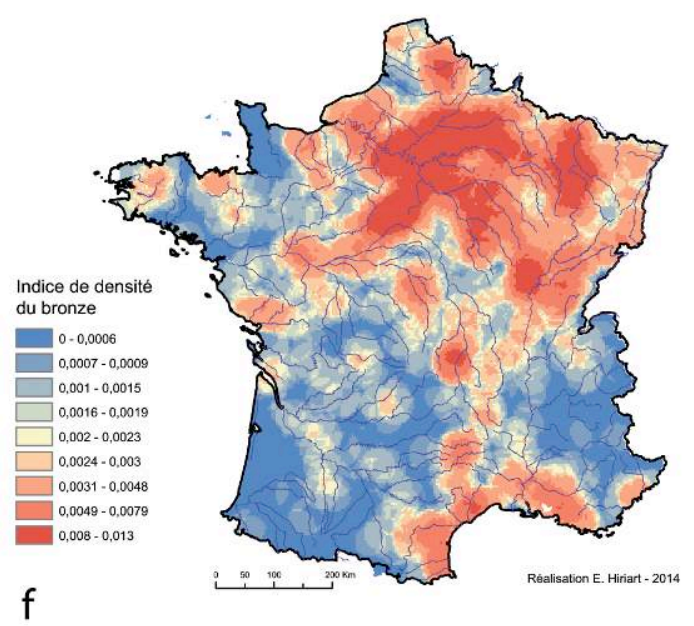

Figure 11 : (Voir planche couleur XVII) Diffusion des alliages monétaires sur les sites III $^{\mathrm{e}}$ s.- $\mathrm{I}^{\mathrm{er}}$ s. av. J.-C. : a) l'or, b) L'argent, c) Le bronze, d) la densité des sites avec de l'or, e) La densité des sites avec de l'argent, f) La densité des sites avec du bronze. K. Gruel, DAO, E. Hiriart. BaseFer mars 2014 ( )

Figure 11: (See colour plate XVII) Distribution of monetary alloys on settlements of III.to I c. BC: a) gold, b) silver c) bronze e) density of settlements with goldf) density of settlements with silver g) density of settlements with bronze. 

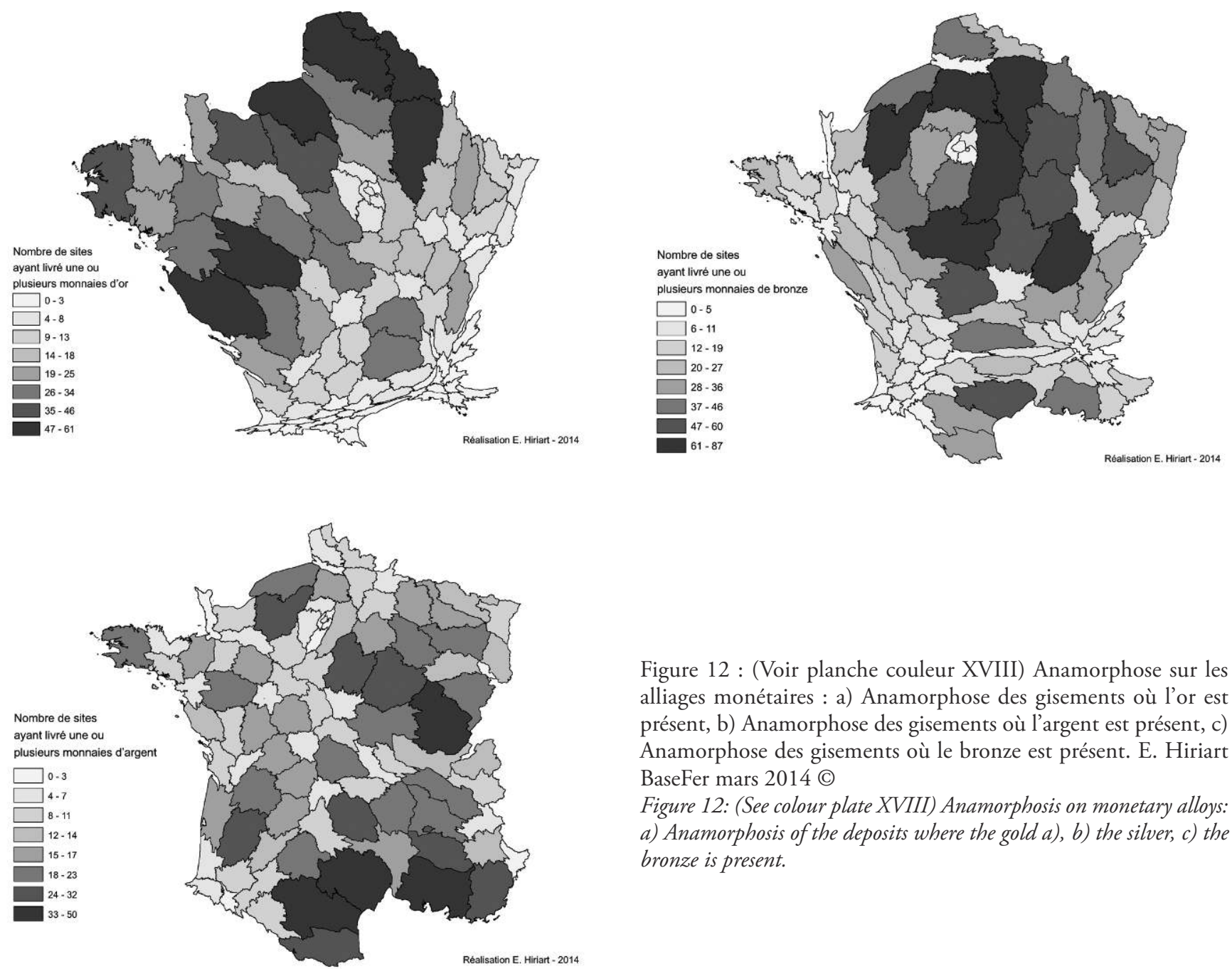

Figure 12 : (Voir planche couleur XVIII) Anamorphose sur les alliages monétaires : a) Anamorphose des gisements où l'or est présent, b) Anamorphose des gisements où l'argent est présent, c) Anamorphose des gisements où le bronze est présent. E. Hiriart BaseFer mars 2014 (C)

Figure 12: (See colour plate XVIII) Anamorphosis on monetary alloys: a) Anamorphosis of the deposits where the gold a), b) the silver, c) the bronze is present.

sujet à un étudiant ou de développer un programme collectif. Enfin les nombreux traitements disponibles aujourd'hui en analyse spatiale, utilisés de plus en plus pour interpréter les masses de données disponibles (Nakoinz, 2005, 2013 ; Gandini, 2008), trouveront là matière à s'exercer. Nous invitons tous les chercheurs qui le désirent à nous contacter pour alimenter et consulter cette base.

\section{Bibliographie}

Batardy C., Buchsenschutz O., Dumasy F. (dir.), 2001. Le Berry antique : atlas 2000, Tours, RACF, 190 p., (Supplément à la Revue archéologique du centre de la France; 21).

Batardy Chr., Buchsenschutz O., Gruel K., Levéry M., 2008. "Analyse spatiale et statistique de l'Âge du Fer en France.

L'exemple de la BaseFer ", Revue Archéologique du centre de la France, Tome 47, revues.org.

Blanchet A., 1905. Traité des monnaies gauloises, Paris.

Buchsenschutz O., Gandini C., Maussion A., 2004. L'archéologie à la conquête de l'espace : les "carte à pois " et le poids des cartes, de la Carte archéologique au Système d'Information géographique. Archéométrie, 2003 : colloque du GMPCA, Bordeaux - Pessac - Talence, 16-19 avril 2003, Revue d'archéométrie, 28 : 5-13.

Buchsenschutz O., 2006. L'apport de la cartographie à l'étude des sociétés sans écriture : l'exemple des Celtes de l'Âge du Fer, M@ppemonde, 83 (2006.3), [http://mappemonde.mgm.frl num1 1/articles/art06305.html].

EgGERs H. J., 1951. « Die vergleichende geographisch-kartographische Methode in der UrgeschichtsForschung ", Archaeologia Geographica, 1, 1950-1951, p. 1-3. 
Gandini C., 2008. Des campagnes gauloises aux campagnes de l'Antiquité tardive : la dynamique de l'habitat rural dans la cité des Bituriges Cubi (II s. av. J.-C.-VII s. apr. J.-C.), Tours, FERACF, juin 2008, 511 + CDRom (supplément à la $R A C F, \mathrm{n}^{\circ} 33$ ).

Gruel K., 2006. «Les prototypes des monnaies gauloises : les raisons de leur choix ». In D. Frere, (dir.), De la Méditerranée vers l'Atlantique : aspects des relations entre la Méditerranée et la Gaule centrale et occidentale (VIII ${ }^{\mathrm{e}}-\mathrm{II}^{\mathrm{e}}$ siècle av. J.-C.). Rennes PUR, coll. «Archéologie \& culture ", p. 67-74.

Gruel K. et al., 1990 : "Les monnaies gauloises en Armorique : présence ou absence dans l'habitat ". In A. Duval, J.-P. Le Bihan, Y. Menez (éd.). Les Gaulois d'Armorique : la fin de l'Âge du Fer en Europe tempérée. XII ${ }^{e}$ colloque de l'AFEAF, Quimper, 1988. Rennes, Association pour la diffusion des recherches archéologiques dans l'ouest de la France, p. 64-69.

Gruel K., Pion P., 2009. Les «trésors monétaires » en Gaule chevelue : faciès régionaux et contextes sociaux des dépôts. In S. Bonnardin, C. Hamon, M. Lauwers, B. Quilliec (éd.), Du matériel au spirituel. Réalités archéologiques et historiques des "dépôts" de la Préhistoire à nos jours, XXIX rencontres internationales d'archéologie et d'histoire d'Antibes. Antibes, Éditions APDCA, p. 381-395.
Nakoinz O., 2005. Studien zur räumlichen Abgrenzung und Strukturierung der älteren Hunsrück-Eifel Kultur, (UPA, 118), Bonn, R. Habelt.

Nakoinz O., 2013. Archäologische Kulturgeographie der ältereisenzeitlichen Zentralorte Südwestdeutschlands, Universitätsforsch. prähist. Arch., 224, Bonn.

Ordnance Survey, 1962. Ordnance Survey map of southern Britain in the Iron Age, Chessington, Surrey, The Survey.

Olmer F. et al., 2013. Voies, acteurs et modalités du grand commerce en Europe occidentale. In A. Colin, Fl. Verdin (éds), L’Âge du Fer en Aquitaine et sur ses marges. Mobilité des hommes, diffusion des idées, circulation des biens dans l'espace européen à l'Âge du Fer: actes du XXXV' Colloque international de l'AFÉAF, (Bordeaux, 2-5 juin 2011), (Aquitania, Suppl. 30), Pessac, Fédération Aquitania, p. 665-691.

Provost M., Carte archéologique de la Gaule, Pré-inventaire archéologique, 128 volumes publiés de 1988 à 2012, coédité par l'Académie des Inscriptions et Belles-Lettres et la Maison des Sciences de l'Homme, Paris. La tomaison renvoie au $n^{\circ} \mathrm{du}$ département auquel l'ouvrage est consacré. 


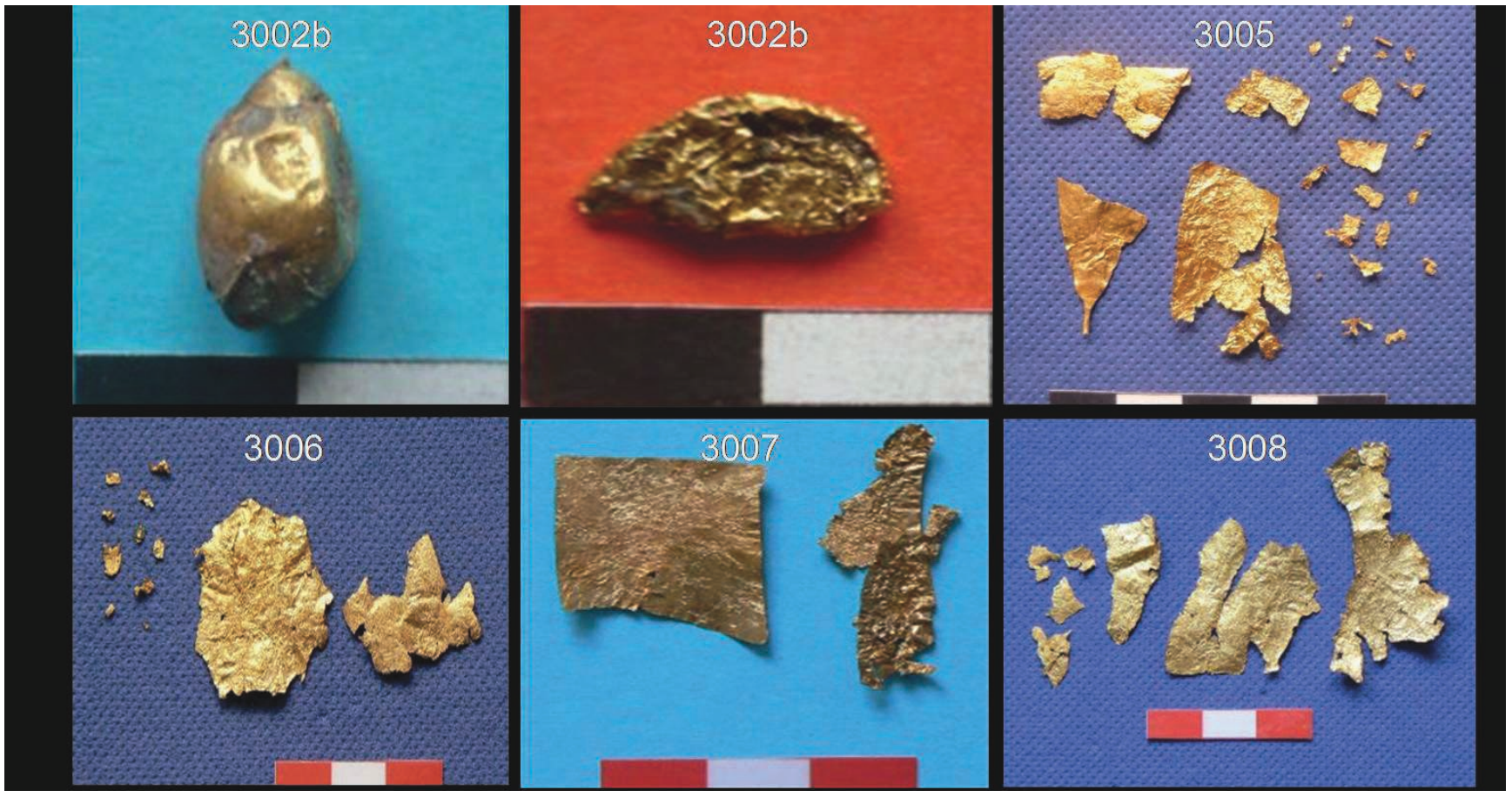

Figure 4 : Deyan Lesigyarski et al., Thracian Golden Wreath from Kabyle, Bulgaria: Chemical Composition (p. 151)

Figure 3 : Olivier BuchsensCHUTZ et al., Une base pour l'élaboration de modèles de peuplement de l'Âge du Fer en France (p. 163)

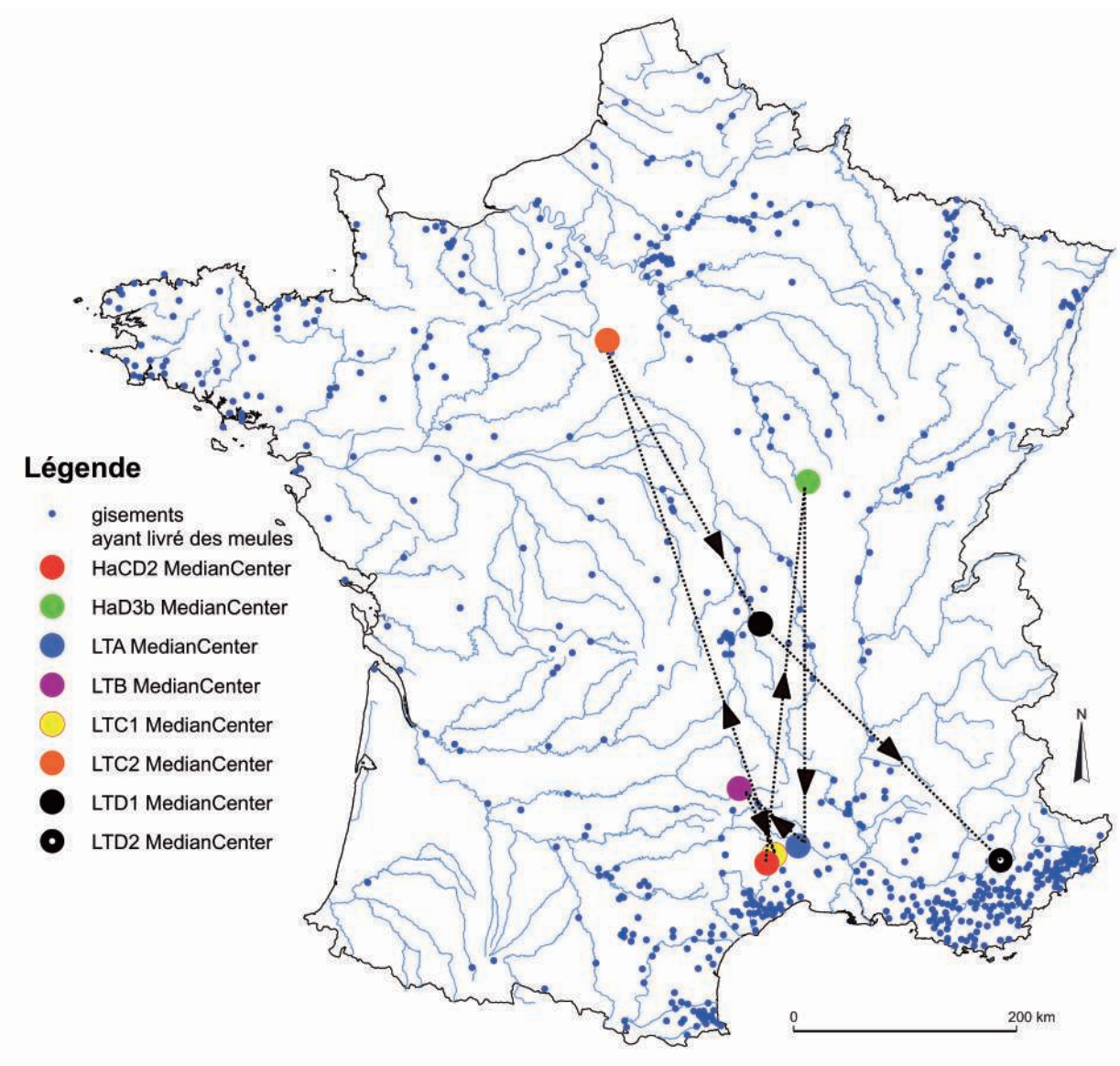

Evolution chronologique du centre médian de la répartition des meules 


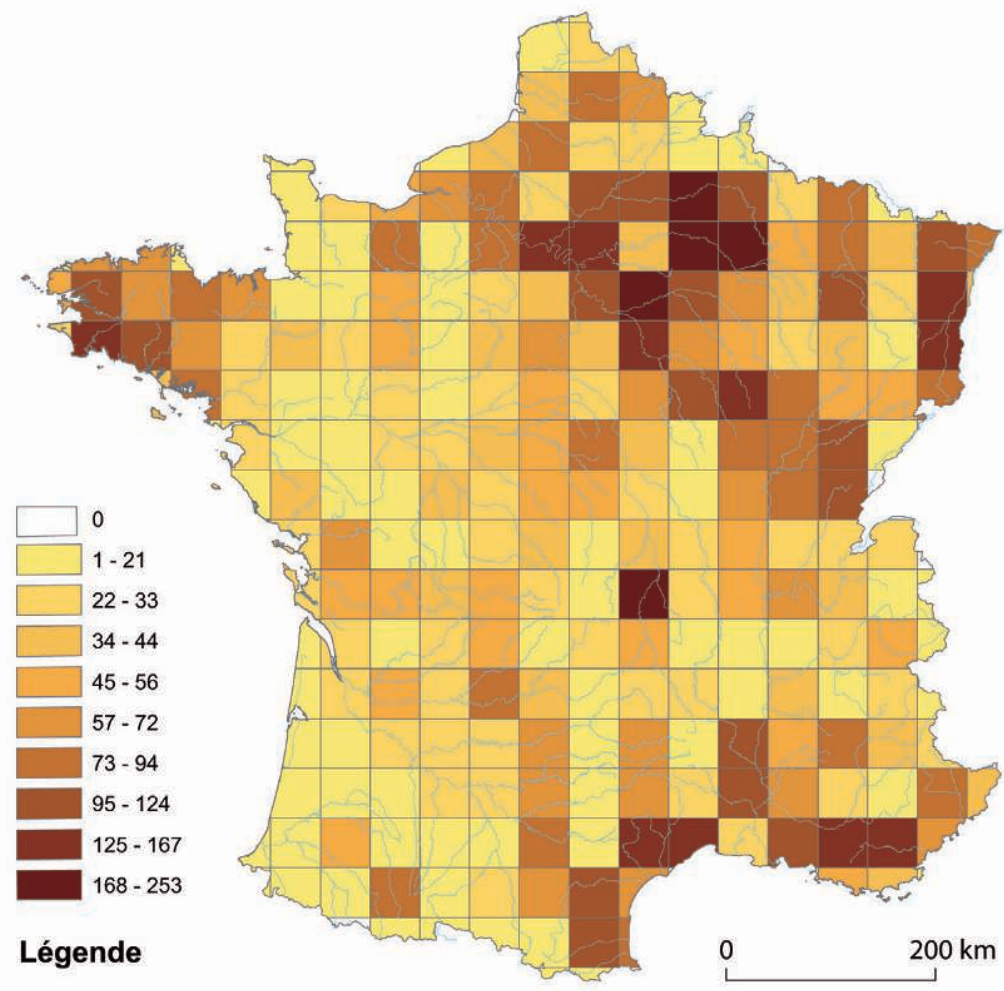

Nombre de gisements par carré de $50 \times 50$ km
Figure 4 : Olivier Buchsenschutz et al., Une base pour l'élaboration de modèles de peuplement de l'Âge du Fer en France (p. 163)
Figure 5 : Olivier BuchsenschuTz et al., Une base pour l'élaboration de modèles de peuplement de l'Âge du Fer en France (p. 164)

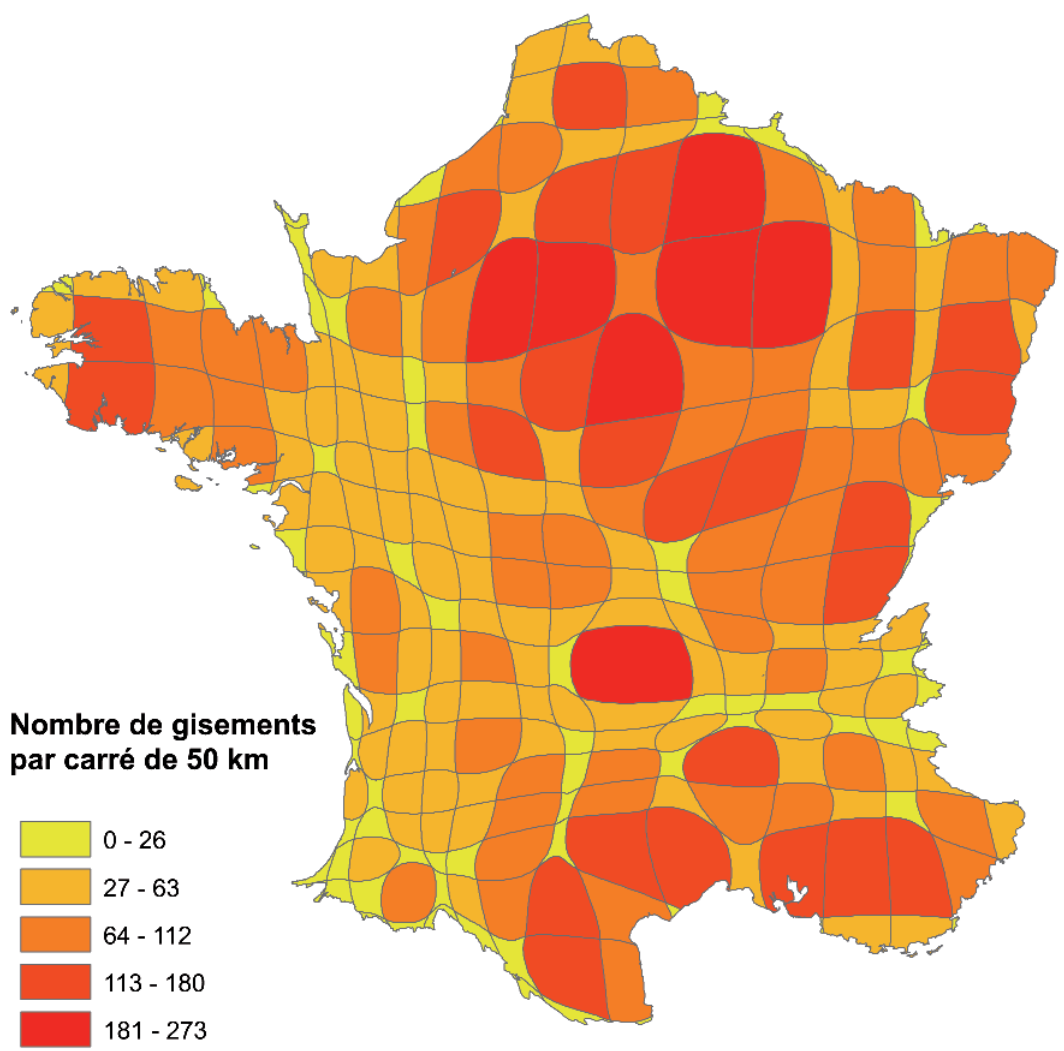




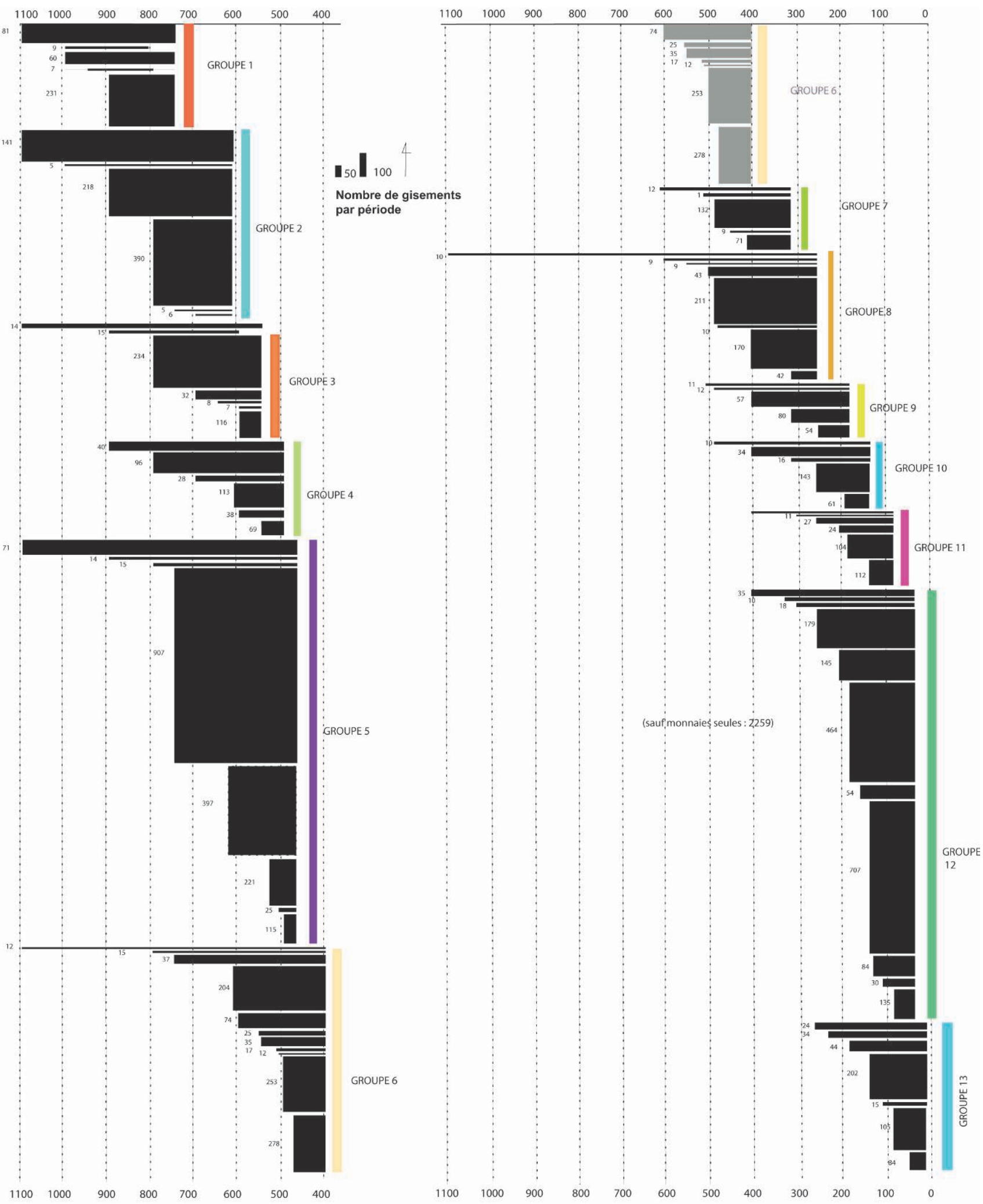

Figure 6 : Olivier Buchsenschutz et al., Une base pour l'élaboration de modèles de peuplement de l'Âge du Fer en France (p. 165) 

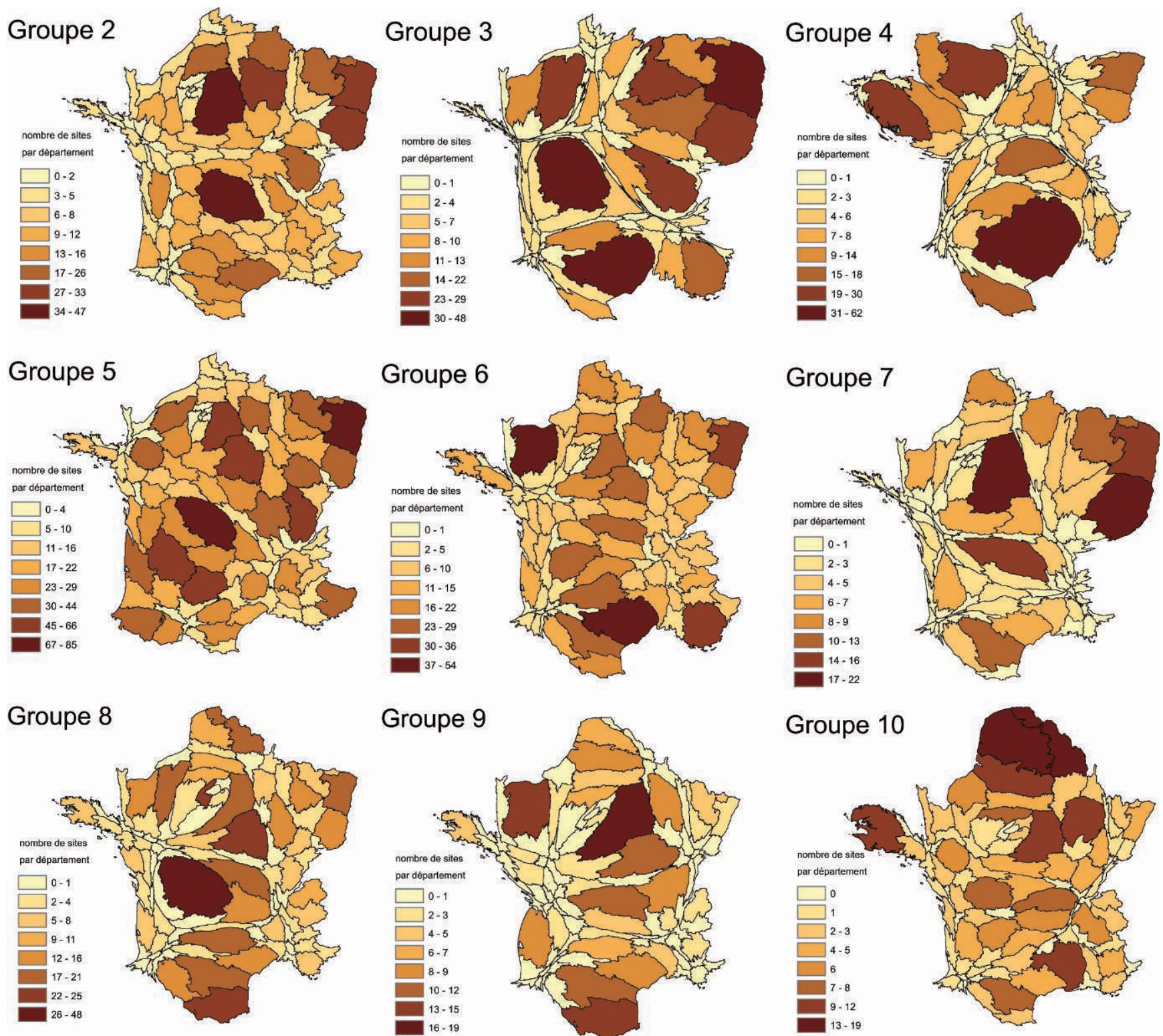

Groupe 9
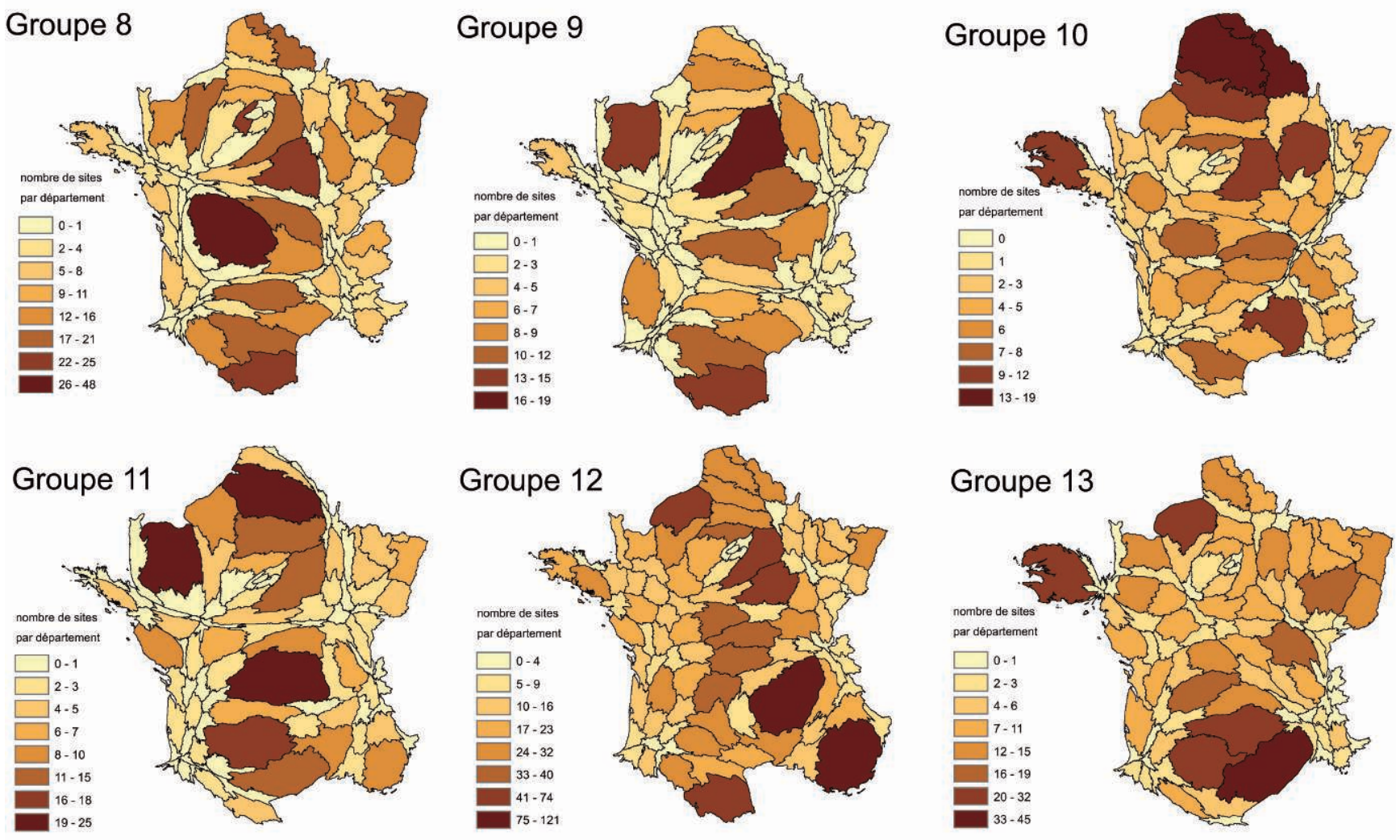

BaseFer mars 2014 ㄷ E. Hiriart

Figure 8 : Olivier Buchsenschutz et al., Une base pour l'élaboration de modèles de peuplement de l'Âge du Fer en France (p. 167) 

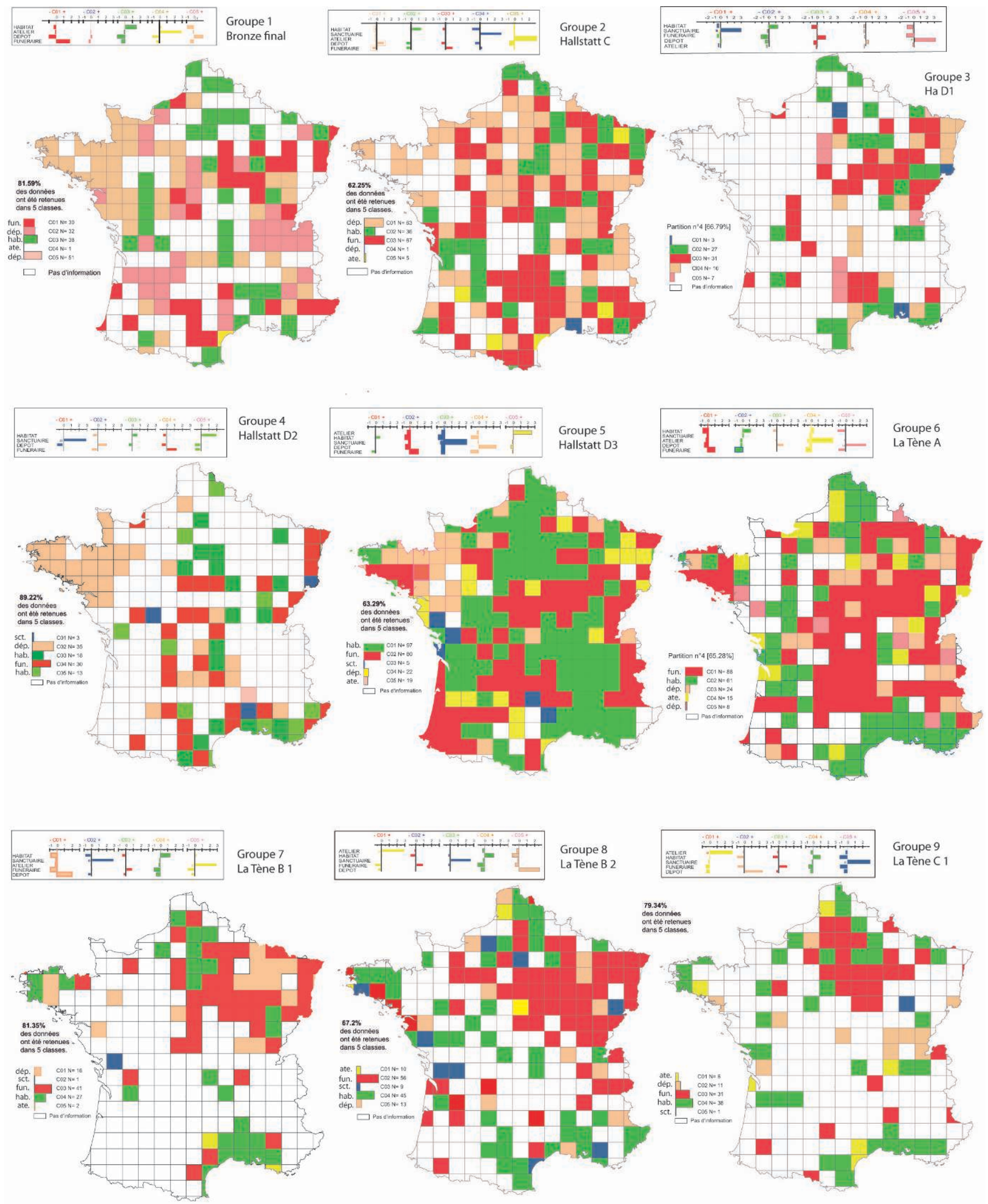

Fait avec Philcarto * 06/10/2014 Ch. Batardy 

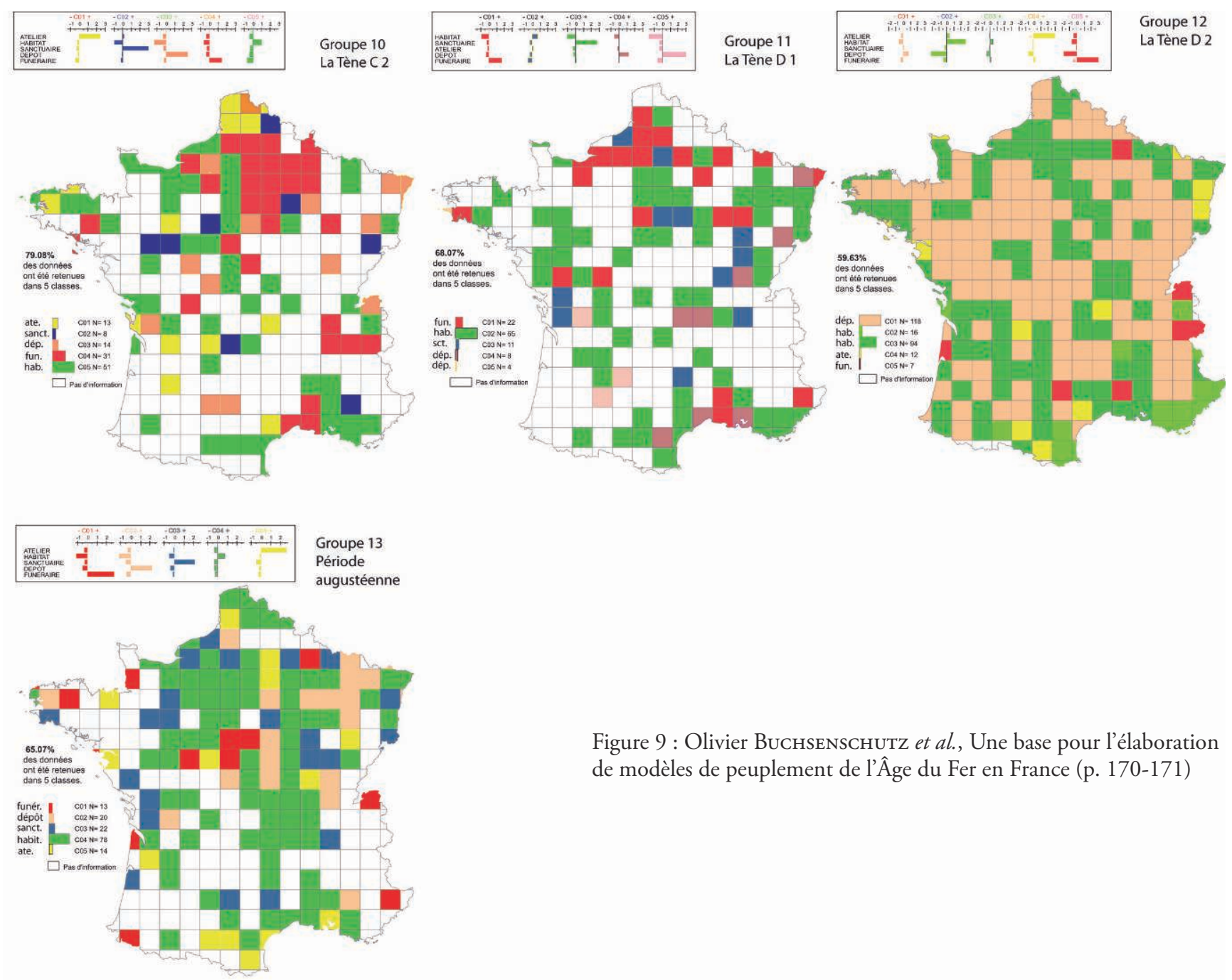

Figure 9 : Olivier Buchsenschutz et al., Une base pour l'élaboration de modèles de peuplement de l'Âge du Fer en France (p. 170-171)

Fait avec Philcarto 06/10/2014 Ch. Batardy 

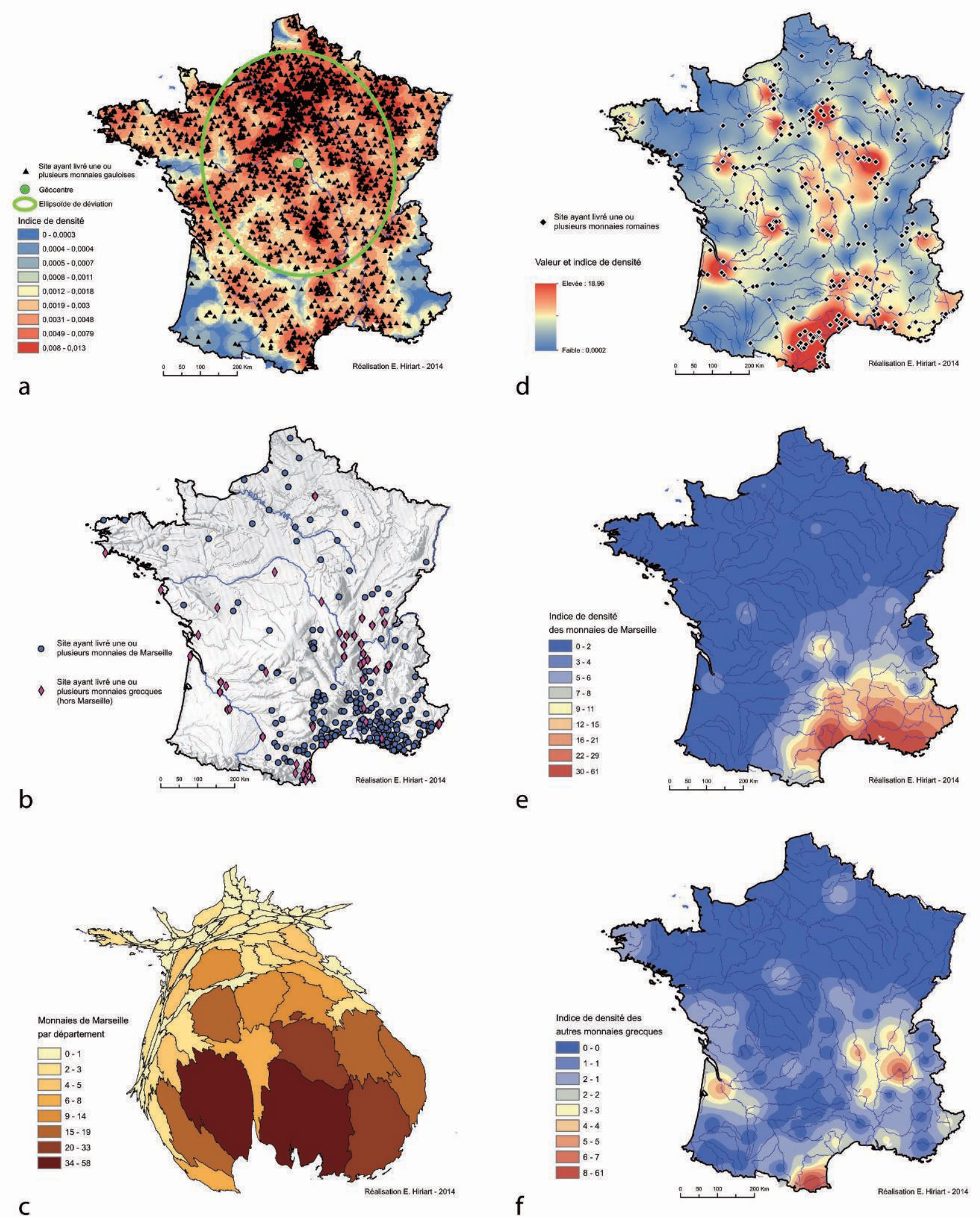

Figure 10 : Olivier Buchsenschutz et al., Une base pour l'élaboration de modèles de peuplement de l'Âge du Fer en France (p. 172) 


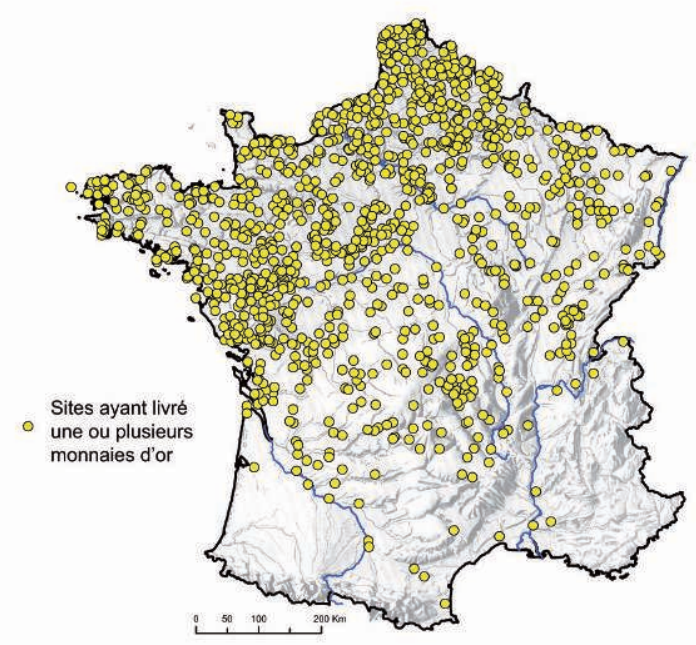

a

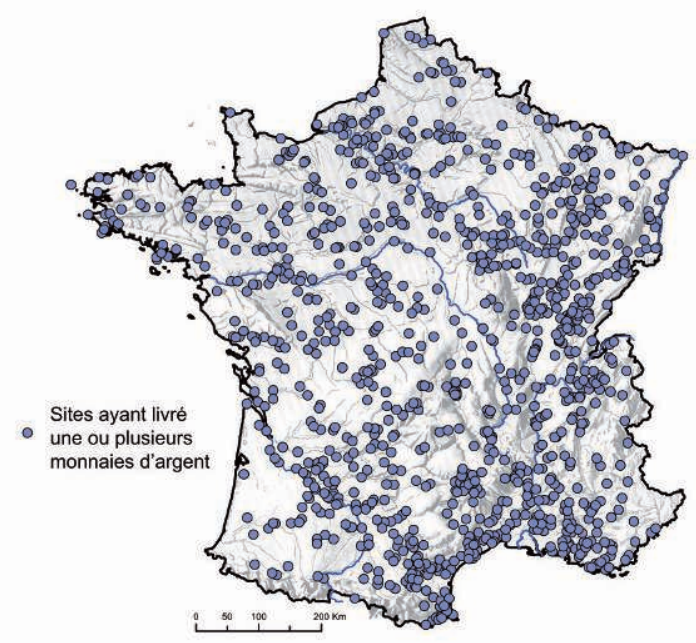

b

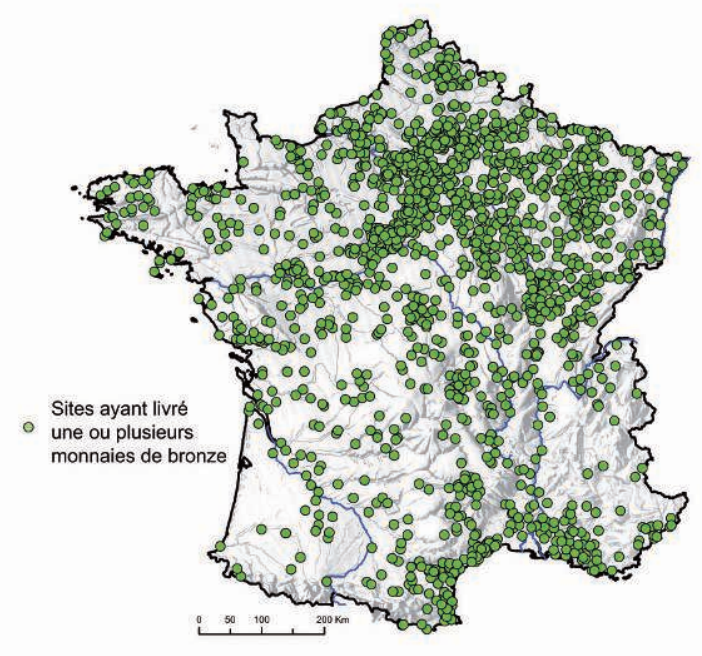

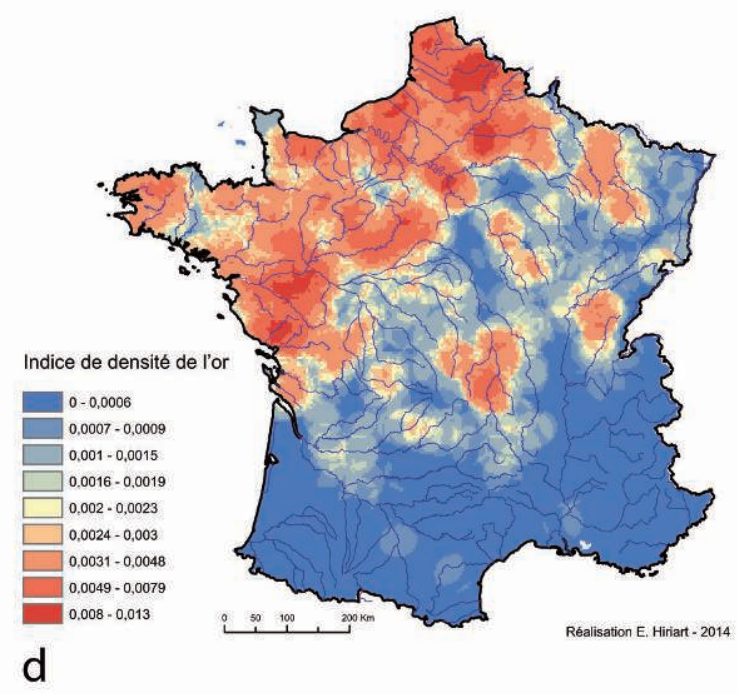
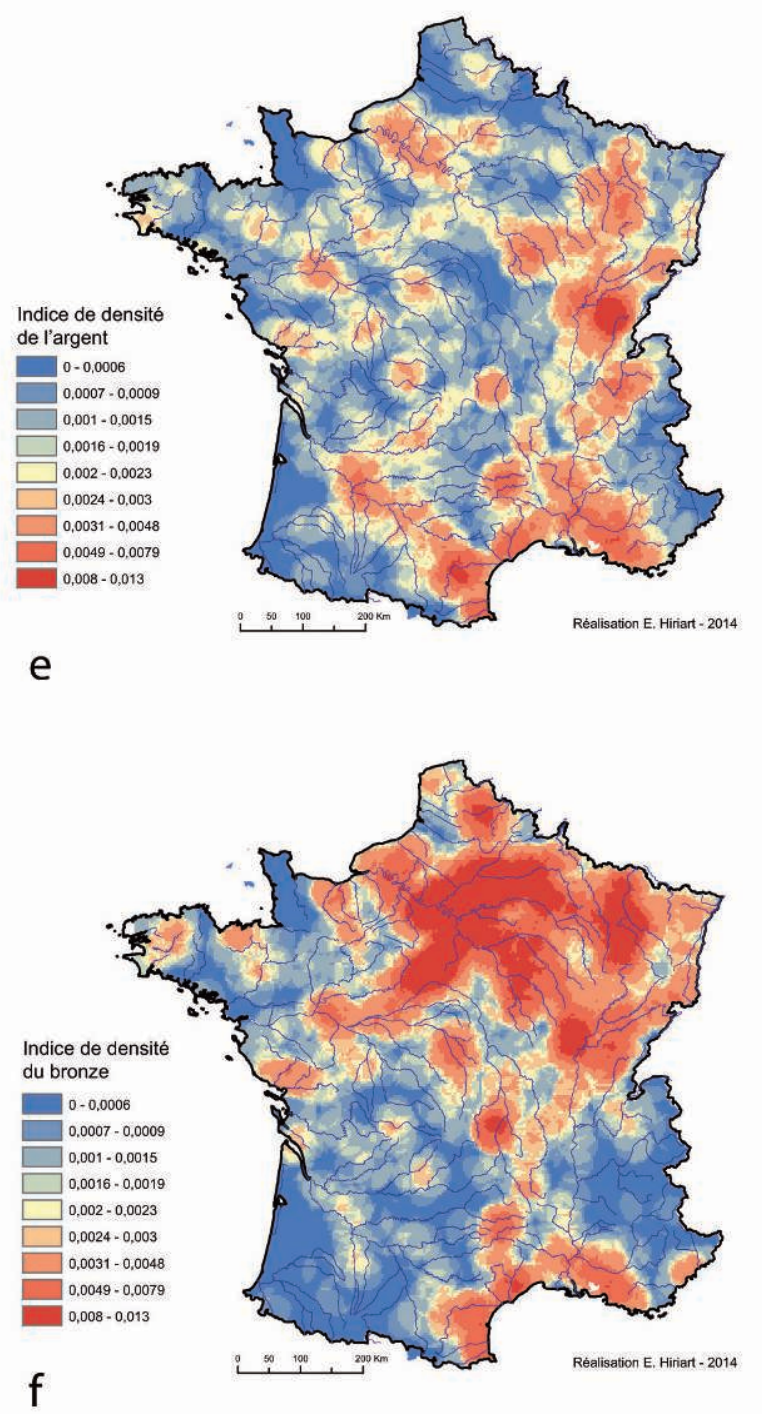

Figure 11 : Olivier Buchsenschutz et al., Une base pour l'élaboration de modèles de peuplement de l'Age du Fer en France (p. 172) 

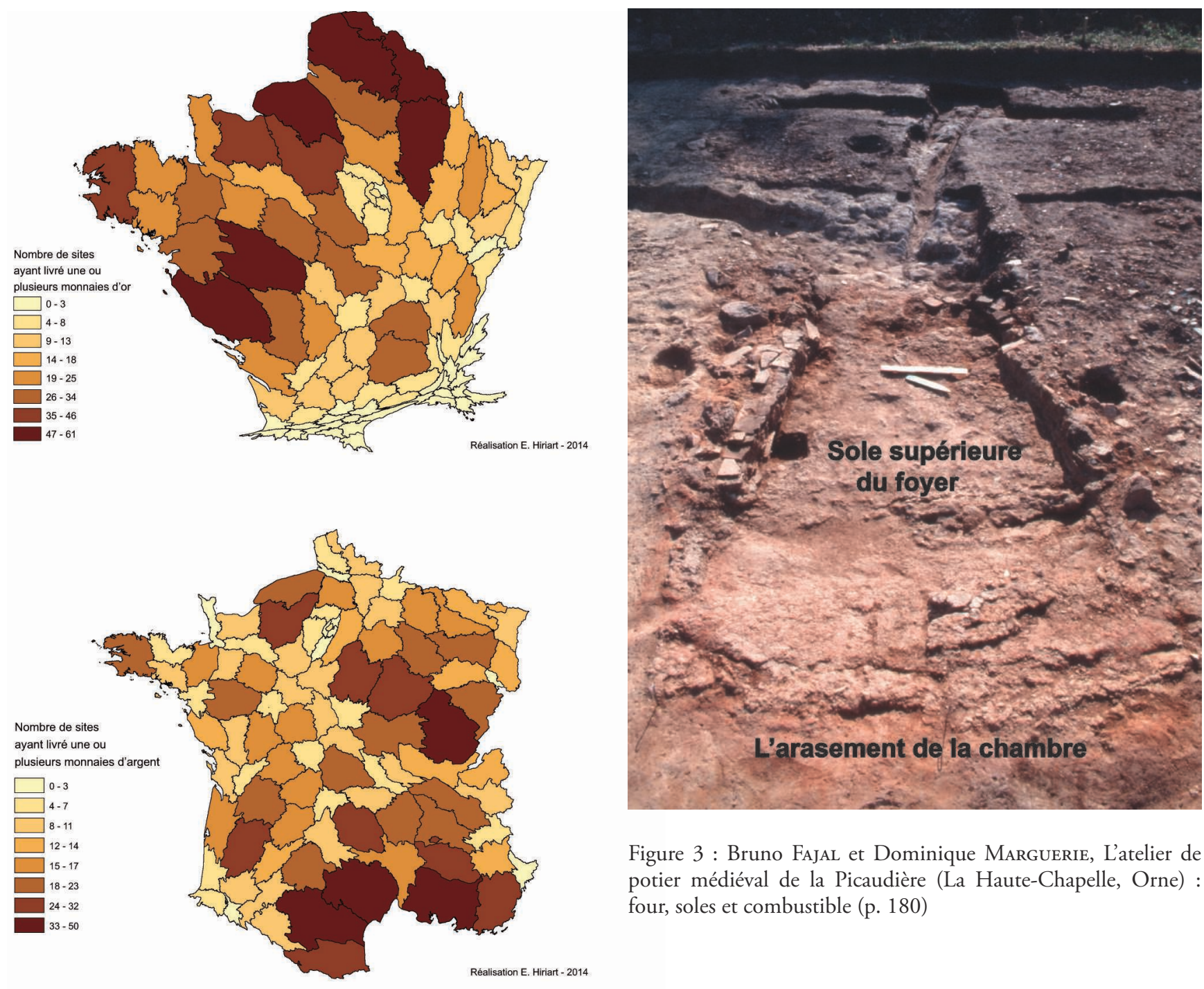

Figure 3 : Bruno Fajal et Dominique Marguerie, L'atelier de potier médiéval de la Picaudière (La Haute-Chapelle, Orne) : four, soles et combustible (p. 180)

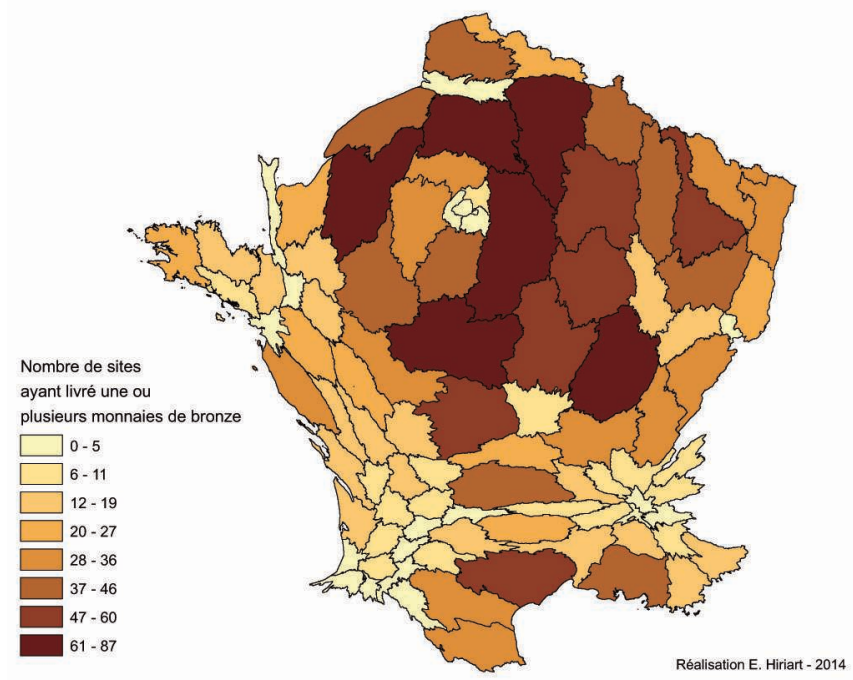

Figure 12 : Olivier Buchsenschutz et al., Une base pour l'élaboration de modèles de peuplement de l'Âge du Fer en France (p. 174) 